\title{
CONDANNA ED ESECUZIONE DI GESÙ: TESTIMONIANZE E PROVE
}

\section{PIERO MARIO GILARDI ${ }^{1}$}

RESUMEN: El propósito de este artículo es hacer una revisión sobre los últimos días de Jesús de Nazaret, ofreciendo un panorama general del mundo en que nació, vivió y murió, pero también analizando los personajes que participaron en su juicio, el proceso que enfrentó, su condena y los efectos que ésta tuvo. Se considera también en este estudio, el papel que ha tenido el Sudario de Turín como testimonio documental para confirmar la credibilidad de los evangelistas al describir el proceso de crucifixión y la muerte de Jesús.

Palabras clave: Fuicio de Jesús, crucifixión, Sudario de Turín, Sanedrín.

ABSTRACT: This article aims to examine the last days of Jesus of Nazareth, first outlining the world in which he was born, lived and died, and later analyzing the characters involved in his trial, the process he faced, the condemnation and its effects. It is also considered the role of the Turin Shroud as a documentary testimony to confirm the credibility of the evangelists in describing the process of crucifixion and death of Jesus.

Key words: Trial of Jesus, crucifixion, Turin Shroud, Sanhedrin.

Sumario: I. La Palestina romana; II. "Ius gladii" ed il "crimen maiestatis"; III. I personaggi del processo; IV. Arresto di Gesù ed interrogatorio dei Sommi Sacerdoti; V. Il giudizio del magistrato romano; VI. Esecuzione della condanna; VII. Le fonti; VIII. La Sindone e la sua storia; IX. Raffronti tra Sindone e narrato evangelico; X. La scienza dove l'occhio non può vedere; XI. Il falso mito del radiocarbonio; XIII. Calcolo probabilistico.

1 Università degli Studi dell'Insubria. Dipartimento di Diritto, Economia e Culture. 


\section{Praefatio}

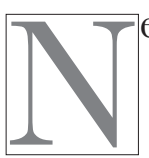

ello scorrere dei secoli innumerevoli studiosi, storici, giuristi, sia credenti di diverse religioni che agnostici od atei, si sono cimentati nella ricerca e descrizione di Gesù, sotto ogni prospettiva ed in ogni suo aspetto. Questa ricerca si è accentuata negli ultimi decenni, non sempre, però, conservando un'impronta equilibrata e scevra da pregiudizi od ideologie, ma spesso volendo adeguare alla proprie opinioni personaggi, fatti e conseguenze, con affermazioni o negazioni apodittiche o pregiudiziali.

Senza tener conto della gran massa di studi che si sono concentrate sull'aspetto trascendente ed escatologico della figura di Gesù, parte delle opere di ricerca si sono appuntate sulla prospettiva storica della vicenda narrata dai Vangeli. Altre, invece, hanno affrontato gli aspetti giuridici, evidenziando radicali divisioni nella ricerca delle responsabilità di ebrei e romani.

Nei limiti consentiti da questo lavoro, l'intento è quello di collocare una tessera nel mosaico della vicenda processuale dell'uomo Gesù, condannato e giustiziato nella Giudea Romana intorno al 30 d.C.: "l'ebreo marginale" dell'opera di un importante studioso dei nostri giorni. ${ }^{2}$ Questo, evidenziando le prove emerse a distanza di quasi due millenni, spesso ignorate od appena accennate, che forniscono veridicità scientifica ad un avvenimento da sempre oggetto di contrapposizioni. Ne è origine l'interesse per uno straordinario "documento", dal quale sempre più la scienza trae conferme e convinzioni: la Sindone. Dalle risultanze di decenni di studi multidisciplinari, ma anche di contestazioni spesso non provate o speciose, l'intento di verificare se quanto la Sindone, vero documento testimoniale, suggerisce, corrisponde a quanto successo al condannato e crocifisso Gesù della narrazione evangelica.

2 J. P. Meier, Un ebreo marginale: ripensare il Gesù storico, Brescia, 2008. 
Il percorso di questo lavoro segue gli ultimi giorni dell'ebreo Gesù di Nazareth, delineando prima il mondo in cui è nato, vissuto e morto, ed analizzando in seguito i personaggi coinvolti nel processo da lui subito, lo svolgimento processuale, la condanna ed i suoi effetti.

Verrà infine esaminato il ruolo di vero e proprio documento testimoniale della Sindone, verificando prima la sua autenticità storica, indi i risultati della straordinaria ricerca scientifica svolta, cose che attestano e confermano l'attendibilità degli evangelisti nel descrivere, pur nella loro scarsa preparazione giuridica e scientifica, la vicenda processuale, la condanna e la morte di Gesù.

\section{La Palestina romana}

A seguito della guerra civile scoppiata alla morte di Gianneo, re della Giudea, Pompeo interviene nel 67 a.C. nella lotta tra i figli; dalla Siria occupa la Palestina e di conseguenza la Giudea, e conquista Gerusalemme. Da quel momento tutta la Palestina passa sotto il controllo politico e militare di Roma. Con accortezza Roma consente la permanenza sacerdotale, lascia autonomia nei riti e libertà religiosa, ed insedia Ircano II, uno dei figli di Gianneo, col titolo di etnarca. ${ }^{3}$ Alla sua morte, nel 43 a.C., sale al potere il figlio Erode, nominato tetrarca. Erode è cinico ed astuto e gode della fiducia dei romani, dai quali farà educare due figli a Roma. ${ }^{4}$ Nel 39 a.C. viene nominato re dei Giudei su proposta di Antonio ed Ottaviano. ${ }^{5}$ Fornisce Gerusalemme di un acquedotto, costruisce una seconda cinta muraria, raddoppia le dimensioni della spianata del tempio, che provvede ad impreziosire ed ampliare, edifica Cesarea Marittima

3 A. Momigliano, Enciclopedia italiana Treccani, p. 193."Etnarca: capo di un popolo orientale al quale, nel mondo romano, viene attribuito rango inferiore a quello di re, ma superiore a quello di Tetrarca".

4 G. Prause, Erode il grande, Milano, 1981, pp. 84, 87.

5 T. Mommsen, L'impero di Roma, vol. VI, pp. 233, 243. 
ed il suo porto. ${ }^{6}$ Vipsanio Agrippa, grande condottiero e genero di Ottaviano, riconoscerà in lui un valido coadiutore nell'opera di ordinamento dell'impero, e come tale Erode verrà sempre trattato. ${ }^{7}$

Oltre agli indiscutibili meriti, Erode è passato alla storia per gli eccessi nella vita privata e la crudeltà nella vita pubblica. Intorno al 5 a.C. a.C. fa sgozzare a Betlemme decine di bambini, dai quali vede minacciato il suo trono, ed è in questo periodo che si colloca la nascita di Gesù. Muore nel 4 a.C., pochi giorni dopo aver fatto uccidere il figlio Antipatro, in precedenza designato al trono. ${ }^{8}$ Gli succedono Erode Antipa quale re di Galilea ed il fratello Archelao come re della Giudea, che però verrà deposto nel 6 d.C. da Augusto. La Giudea diviene così provincia romana speciale. ${ }^{9}$

Riguardo all'amministrazione della giustizia in Giudea, Roma conferisce al Sinedrio ed al Sommo Sacerdote la giurisdizione civile sui giudei, in quanto i diritti romani non vengano lesi. Per i cittadini romani, invece, la giurisdizione è del governatore e coesiste con quella dei magistrati di Roma, ai quali il governatore può sempre indirizzare il richiedente. Resta fermo il diritto di avocazione da parte del princeps. ${ }^{\circ}$ Circa la giurisdizione criminale, il diritto di condannare a morte spetta all' imperatore in forza del suo imperium maius et infinitum, diritto che può demandare ai governatori con delega ampia e discrezionale. Tale potere non è però esercitabile sui cittadini romani, che godono del diritto di provocatio, cioè di esser giudicati a Roma. ${ }^{11} \mathrm{Al}$ Sinedrio resta il solo potere residuo di con-

${ }^{6}$ G. Prause, Erode...,op.cit., p. 85.

7 T. Mommsen, L'impero...op.cit., p.243.

${ }^{8}$ G. Ricciotti, Vita di Gesù Cristo, Milano, 1999, p.78; Giuseppe Flavio, Antichità giudaiche 17, 191.

9 T. Mommsen, L'impero...op.cit., p.248.

10 T. Mommsen, Le droit public romain, tome III, Paris, p. 308; L'impero...op.cit., pp. 233, 240.

11 Cassio Dione, Storia di Roma, 64, 2, cita il caso di Capito, governatore della Bassa Germania, condannato a morte da Galba per non aver deferito all'impera- 
dannare a morte e di eseguire la sentenza nei confronti di stranieri che varchino la balaustra del Santuario. ${ }^{12}$

Gran parte della dottrina è stata a lungo unanime nell'individuare in procurator il governatore della Giudea, e l'indicazione di Pilato con questa qualifica ancora persiste, sulla base dei racconti evangelici, di quanto affermato da Giuseppe Flavio e soprattutto da Tacito $^{13}$. Ma Tacito scrive circa ottant'anni dopo gli avvenimenti descritti, quando la qualifica di procuratore è diventata da tempo ufficiale sotto Claudio. Però una lapide rinvenuta nel 1961 a Cesarea, contenente una dedica di Pilato a Tiberio, conferma senza alcun dubbio che il governatore della Giudea aveva il titolo di Praefectus, ${ }^{14}$ e con esso l'imperium militare pro legatum. Sarà solo in seguito, con Claudio, che i governatori assumeranno il titolo di Procurator.

II. IL "IUS GLADII" ED IL "CRIMEN MAIESTATIS"

Il ius gladii è identificabile col potere punitivo dei governatori sui residenti delle provincie, ed è comprensivo della pena di morte, come suggerisce il nome stesso. E' un potere da esercitare tempestivamente, per evitare che vengano compromessi gli interessi generali. ${ }^{15}$ In Giudea, come detto, viene tolto al Sinedrio il potere di mettere a morte, conservandogli la sola possibilità di occuparsi di reati minori punibili con carcere o fustigazione. Per reati comportanti pena di morte, tranne il caso della balaustra del Santuario, resta al Sinedrio

tore l'appello di un cittadino romano.

12 G. Flavio, Antichità giudaiche, 15, 11, 5

13 G. Flavio, La guerra giudaica, 2, 8, 1; Antichità...op.cit., 18, 1, 1; Tacito, Annales 15, 44 "Auctor nominis eius Christus Tiberio imperante per procuratorem Pontius Pilatum supplicio adfectus erat".

14 A. Degrassi, Sull'iscrizione di Ponzio Pilato, in Rend.Lincei, Roma, 1964, p. 60. "[...]S TIBERIEUM[...] NTIUS PILATUS[...] EGTUS IUDAE. E".

15 V. Campolunghi, BIDR 75 (1972), p. 210; A.Manfredini, Ius gladii, Annuario Univ. Ferrara, vol. V, p. 119 ss. 
il solo compito istruttorio, senza il potere di emettere sentenze. ${ }^{16} \mathrm{E}$ ' un divieto mal sopportato, testimoniato dalla brutale esecuzione di Stefano: non potendolo condannare a morte viene trascinato fuori e lapidato: ${ }^{17}$ si tratta però di un'esecuzione sommaria, che non implica deroghe alla proibizione generale.

Riguardo al crimen maiestatis, nell'antica Roma la maiestas era attributo dello stato romano e col reato di laesa maiestatis veniva punito, secondo Cicerone, «ogni atto volto a sminuire, disconoscere o derogare la dignità, l'ampiezza od il potere del popolo romano, ovvero di coloro ai quali il popolo ha conferito il potere». ${ }^{18}$ Dall' 8 a.C., con la lex Iulia maiestatis di Augusto, verranno puniti non solo i reati contro lo stato ma anche quelli contro la persona e la sacralità dell'imperatore, personificazione dello stato. Con Tiberio crimen maiestatis diverrà pure qualunque offesa al ruolo del princeps, commessa sia da funzionari sia da privati. ${ }^{19}$

Nel processo a Gesù la principale accusa riguarderà l'affectatio regni, cioè l'essersi qualificato re e quindi mirare alla costituzione di un potere monarchico e, di conseguenza, ambire a spodestare l'imperatore. Si tratta di comportamenti che rientrano a pieno titolo nel reato di crimen maiestatis.

\section{I PERSONAGgI DEL PROCESSO}

Il percorso non può che iniziare con la figura di Gesù, protagonista dei fatti che gli sono costati una condanna infamante e crudele. Verrà poi tratteggiata la figura di Ponzio Pilato, praefectus della Giudea, e quella del Sinedrio e dei Sommi Sacerdoti, espressione del potere

16 M. Miglietta, I. N. R. I., Napoli, 2011, p. 32. Contra: J. Blinzler, Il processo di Gesù, Brescia, 1966, p. 198.

17 Atti, 7, 57-58.

18 Cicerone, De inv. Reth, 2,17,53 "Maiestatem minuere est de dignitate aut amplitudine aut potestate populi romani aut eorum, quibus populus potestatem dedit, aliquid derogare".

19 A. Guarino, Storia del diritto romano, Napoli, 2001, pp. 280, 422, 443. 
della classe dominante sadducea in tema di fede, costumi e regole civili in Giudea e gran parte di Palestina. La vicenda di ciascuno d'essi è accomunata da un destino terreno terminato brutalmente: uccisione per Gesù; condanna a morte o suicidio per Pilato; distruzione di Gerusalemme, crollo del potere sinedrile e dispersione dei giudei. Ma il percorso comune termina qui: di Gesù si sono occupati e si occupano studiosi di ogni branca, ed innumere persone di cui è stato od è riferimento di vita, oppure oggetto di dubbi od avversione; degli altri, tanto potenti da segnare un'epoca, il destino di popolazioni e la vita stessa di Gesù, è solo la storia ad occuparsene, spesso di riflesso alla vicenda terrena di quell' ebreo marginale del primo secolo.

Sul luogo di nascita di Gesù i pareri sono discordi: Betlemme in Giudea per alcuni, Nazareth in Galilea per altri. Chiarire l'origine ha significato per comprendere le discussioni sulla competenza fra Pilato, Erode Antipa ed il Sinedrio. Secondo Matteo Gesù nasce a Betlemme, la città di Davide alla cui casata sarebbe appartenuto; ${ }^{20}$ aggiunge poi che la famiglia si trasferisce a Nazareth dopo la fuga in Egitto. ${ }^{21}$ Luca fornisce un preciso chiarimento: si tratta di Betlemme, città della Giudea e luogo di origine della famiglia di Giuseppe, padre di Gesù, ivi recatosi per sottoporsi al censimento voluto da Augusto «quando Quirinio era governatore della Siria»". ${ }^{22}$ Marco e Giovanni non parlano direttamente della nascita di Gesù.

Alcuni studiosi ed autori, pur citando espressamente Matteo e Luca, situano viceversa la nascita a Nazareth, basandosi sul fatto di essere Gesù chiamato Nazareno o di Nazareth. ${ }^{23}$ Le spiegazioni, spesso contraddittorie, appartengono al campo delle supposizioni, e

20 Matteo, 2,1 "Nato Gesù in Betleem di Giuda, al tempo di re Erode".

21 Idem, 2, 19-23 "Giuseppe [...] si ritirò nel territorio della Galilea, ed andò ad abitare in una città chiamata Nazaret".

22 Luca 2, 1-7.

23 Tra i più recenti: D. Flusser, Jesus, Brescia, 2008, p. 38; J. P. Meier, Un ebreo... op. cit., p. 38; C. Augias, M. Pesce, Inchiesta su Gesù, Milano, 201 1, p. 10. 
si contrappongono alle precise affermazioni di Matteo ed ancor più a quelle di Luca. Questi è uomo colto, probabilmente medico. Nel suo vangelo, destinato al mondo romano, parla con precisione del primo censimento di Augusto, con Quirino governatore in Siria: è impensabile che si avventuri in citazioni di avvenimenti non veritieri, facilmente smentibili sia a Roma che in Palestina. Né del resto si contraddice quando dice che, durante il processo, Pilato lo invia ad Erode Antipa: non parla di Gesù come nato in Galilea, bensì della giurisdizione di Erode. ${ }^{24}$ E' vero che a Nazareth Gesù ha vissuto molta della sua vita ed ha svolto gran parte della sua predicazione, ma come si giustifica che Erode lo rimandi da Pilato se, oltre che vissuto, fosse anche nato in Galilea e quindi suo suddito? Che sia Pilato a processarlo testimonia che Gesù è riconosciuto giudeo, e che la giurisdizione spetta quindi al praefectus.

Anche la data di nascita di Gesù è discussa. Il nostro calendario è basato sui calcoli di Dionigi il Piccolo, che pone l'anno 1 della nostra era al 753 a.u.c. In realtà è ormai accertato che Dionigi abbia sbagliato i suoi calcoli di 6 anni, cosa che anticipa la reale data di nascita di Gesù al 6 a.C. Questa data è coerente con la strage dei bambini inferiori ai due anni, ordinata da Erode verso il 5 a.C. ${ }^{25}$ e con la sua morte nel 4 a.C.

L'inizio della vita pubblica di Gesù coincide col battesimo ricevuto da Giovanni Battista intorno al 28 d.C. Degli anni precedenti poco o nulla si conosce, salvo dar credito ai vari vangeli apocrifi, non accolti dalla chiesa. La sua vita è probabilmente stata quella di un ebreo vissuto nella famiglia, nel lavoro e nella formazione culturale e religiosa. E' scontato che Gesù parli il dialetto aramaico; sappiamo anche che legge correntemente i testi in ebraico e li discute coi dottori della legge: la sua formazione ebraica è giudicata

\footnotetext{
24 Luca, 23, 5-7.

25 G. Ricciotti, La vita..., op.cit., p. 78.
} 
incomparabilmente più elevata di quella di Paolo. ${ }^{26}$ E' accertato, esaminando la costruzione sintattica delle parabole, che abbia buona conoscenza del greco, ed è probabile che sappia esprimersi in latino, cose queste testimoniate dagli interrogatori di Pilato, difficilmente eseguiti in aramaico od in ebraico. La sua vicinanza e la parentela col Battista (le madri erano cugine) ha fatto ad alcuni supporre che Gesù fosse simpatizzante esseno o patriota zelota, ma ciò non trova alcun riscontro nei vangeli, nello svolgimento del processo, e neppure in Giuseppe Flavio. ${ }^{27}$

Al momento del battesimo Gesù ha circa trentaquattro anni: tenuto conto che la sua predicazione ed il peregrinare tra Galilea e Giudea durano poco più di due anni, la sua opera si interromperà bruscamente all'età di circa 36 anni, alla vigilia della Pasqua del 30 d.C., probabilmente il venerdì 7 aprile.

Il governatore della Giudea è Ponzio Pilato. Risiede di norma a Cesarea Marittima, salvo trasferirsi a Gerusalemme, nel palazzo già di Erode il Grande, in occasione di festività o per necessità connesse al suo incarico di praefectus. Si ipotizza una sua nascita intorno al 16 a.C. da una famiglia di probabile origine sannitica. Di lui nulla si conosce sino alla sua nomina nel 26 d.C. a prefetto della Giudea, carica che manterrà sino al 36-37 d.C., quando verrà richiamato a Roma per il suo comportamento e per abusi nei confronti di alcuni samaritani. ${ }^{28} \mathrm{Da}$ allora si perdono le sue tracce: chi lo dice condannato da Caligola, chi suicida, chi morto annegato. Le fonti storiche coeve su Pilato sono i vangeli, la lettera di Erode Agrippa a Caligola, gli Atti degli Apostoli e le lettere di S.Paolo. Quelle immediatamente posteriori sono Giuseppe Flavio e Tacito. ${ }^{29}$

26 D. Flusser, Fesus, op.cit., pp. 42-43.

27 G. Augias, M. Pesce, Inchiesta..., op.cit. p. 54.55." [...] Nella sua lotta possono esserci la vittoria o la sconfitta, mai il compromesso [...] Per realizzare questo suo scopo ha fatto uso della forza o di strumenti politici? No, non lo ha fatto".

28 G. Flavio, La guerra...,op.cit., 2, 175-179.

29 G. Flavio, Antichità...,op.cit., 18, 63-64; Tacito, Annales, 15, 44. 
Il prefetto Pilato è innanzitutto un comandante militare, investito dell'imperium, della coercitio e del ius gladii, cioè il potere di mettere a morte chi commetta crimini gravi, primo fra tutti il crimen maiestatis. Quanto alla persona ed al suo comportamento, il re Erode Agrippa così lo descrive in una lettera del 40 d.C. indirizzata al suo protettore ed amico Caligola: «Di carattere inflessibile e spietatamente duro. Al suo tempo in Giudea dominavano corruzione, violenza, ruberie, oppressione, umiliazione, continue esecuzioni senza processo e sconfinata intollerabile crudeltà». ${ }^{30}$ Erode forse non è ben disposto ed obiettivo, ma certo la permanenza di Pilato in Giudea è costellata da provocazioni e prove di forza nei confronti degli ebrei e della loro religione, che non comprende e probabilmente disprezza. Con un tale carattere è facile capire perché Pilato mostri tanta riluttanza a condannare e far giustiziare un personaggio ai suoi occhi insignificante come Gesù, ben lontano dall'essere ed anche solo somigliare ad un agitatore politico, cedendo solo al ricatto ed alla minaccia dei giudei e del Sinedrio di denunciarlo all'imperatore per non voler perseguire il reato di affectatio regni e, di conseguenza, il crimen maiestatis.

L'altro protagonista del processo a Gesù è il Sinedrio di Gerusalemme ed il suo massimo esponente, il Sommo Sacerdote Caifa. Il Sinedrio è composto da 71 membri, ed è l'organo preposto a far rispettare le leggi della Torah ed alla gestione amministrativa e della giustizia. Dopo la costituzione della Giudea a provincia, il Sommo Sacerdote che presiede il Sinedrio viene nominato dal prefetto romano. Il Sommo Sacerdote è il capo del Sinedrio, il suo giudice supremo ed il ministro principale del culto del Tempio. Accedere al sommo sacerdozio significa appartenere a famiglie dell'aristocrazia giudaica benestante, ed è noto come la nomina od il rinnovo di questa ambita carica siano oggetto di trattative e mercanteggiamento tra l'interessato ed il prefetto. Dal 6 e fino al 70 d.C., data

3о Filone Alessandrino, De legatione ad Caium, 38, p. 299 ss. 
di distruzione del tempio, il sommo sacerdozio sarà prerogativa di Anna, precedente Sommo Sacerdote e suocero di Caifa, e dei suoi discendenti o parenti: che la famiglia di Anna abbia monopolizzato la carica mostra quale fosse il suo ascendente e la sua capacità economica.

Il Sinedrio è composto da sacerdoti, anziani del popolo e scribi. Questi ultimi sono dottori della legge e vengono scelti tra la classe colta; alcuni di loro, a differenza della classe sacerdotale sadducea, sono farisei. Dei farisei va detto che, pur non facendo parte del Sinedrio se non attraverso quegli scribi che vi partecipano a titolo personale, sono una parte importante della società giudaica. La loro formazione, fatta sia di rispetto della Legge che della tradizione orale, li vede avversari della compromessa classe sacerdotale, ma anche antagonisti e ribelli alla dominazione dei romani, che diffidano di loro.

Una parte del credo farisaico lo ritroviamo nella religione cristiana, e questo ha indotto a credere che Gesù ed i discepoli fossero di estrazione farisaica. Farisei dichiarati sono Paolo, prima della sua drammatica conversione, ed anche Giuseppe d'Arimatea e Nicodemo, che incontreremo al momento della deposizione e sepoltura di Gesù.

iv. Arresto di Gesù ed interrogatorio dei Sommi SACERDOTI

Si colloca al termine della cena pasquale consumata da Gesù coi suoi discepoli, compreso Giuda Iscariota che l'ha tradito accordandosi coi sinedriti. ${ }^{31}$ Né i vangeli né i primi scritti cristiani forniscono una data precisa, sì che gli studiosi hanno dovuto ricorrere ai pochi dati evangelici e storici ed anche all'astronomia. La maggioranza propende per il 30 d.C., tenuto conto che la predicazione del Bat-

31 Matteo, 26, 14-16; Marco, 14, 10-11; Lc., 22, 1-6. 
tista, collocata nel $15^{\circ}$ anno di regno di Tiberio, porta all'inizio del 28 d.C. e che la vita pubblica di Gesù dura circa due anni. In base alle tavole lunari, l'Istituto astronomico di Berlino fissa il venerdì di Pasqua al 7 aprile dell'anno 30 d.C. ${ }^{32}$

Al termine della cena pasquale Gesù ed i discepoli raggiungono il giardino del Getsemani, sul Monte degli Ulivi, ove raccogliersi a pregare. ${ }^{33}$ Qui accade un fatto narrato da Luca che per secoli non ha trovato spiegazione: Gesù nell'intensità della preghiera suda sangue. ${ }^{34}$ Occorre ricordare che Luca è medico e come tale dotato di grande spirito di osservazione sulla fisiologia: quello che descrive è un fenomeno raro, ma noto in medicina col nome di ematidrosi o diapedesi, che può manifestarsi in situazioni di grande stress emotivo, come rilevato nella seconda guerra mondiale su alcuni internati nei lager. ${ }^{35}$

Negli ultimi periodi della sua predicazione Gesù è stato ripetutamente minacciato dai farisei a causa della sua predicazione e, poco prima della Pasqua, il Sinedrio decide di procedere alla sua cattura ed eliminazione ricorrendo al tradimento di Giuda. A fronte dell'indecisione dei farisei, emergono la scaltrezza ed il cinismo del Sommo Sacerdote Caifa, che riesce a far prevalere l'opinione che sia meglio il sacrificio di una persona piuttosto che quello del popolo; infatti il timore è che la sua predicazione provochi l'insorgere delle folle ed il conseguente intervento di Roma, cosa che provocherebbe il crollo del potere sinedrile e suo personale. ${ }^{36}$

La cattura avviene nella notte tra giovedì e venerdì, secondo Marco ad opera «di una gran turba armata, mandata dai sacerdoti,

32 J. Blinzler, Il processo...,op. cit., pp. 85 ss.

33 K. A. Speidel, Il processo a Gesù, Bologna, 1981, p. 68 "Getsemani ha significato di torchio dell'olio".

34 Luca, 22, 43-44.

35 M. Llorrente, 42 giorni, Milano, 1998, p.64.

36 Giovanni, 11, 47-53; J. Blinzler, Il processo...,op. cit., p. 69. 
dagli scribi e dagli anziani, accompagnata da Giuda $;{ }^{37}$ similmente scrivono gli altri sinottici. Solo Giovanni aggiunge la presenza di una coorte romana, cosa che pare strana. Riguardo a questa eventuale presenza, Blinzler la giudica estremamente inverosimile e della stessa opinione è Miglietta, sia per il fatto che i tre sinottici non ne parlano, sia perché la coorte non poteva essere utilizzata senza il consenso di Pilato che, benché in Gerusalemme, stando ai vangeli non sapeva ancora nulla. ${ }^{38}$ Non si può dubitare della legalità formale dell'arresto: le milizie incaricate infatti agiscono al servizio del Sinedrio, arrestano Gesù e lo conducono da Anna, il suocero di Caifa. ${ }^{39}$ I vangeli non dicono quali siano le accuse che giustificano l'arresto di Gesù: si può ipotizzare trattarsi dei reati di blasfemia, falsa profezia e mancato rispetto del sabato.

Ci si chiede perché Gesù venga portato in modo informale da Anna: le ipotesi sono diverse e forse la più verosimile è che Caifa, che nutre rispetto e stima per il suocero, voglia sfruttarne la grande esperienza e scaltrezza per ottenere materiale utile per le successive udienze innanzi al Sinedrio ed a Pilato, utilizzando anche questo lasso di tempo per procedere alla convocazione della corte sinedrile. L'interrogatorio da parte di Anna ha carattere del tutto irrituale e si può considerare una sorta di udienza preliminare (istituto ignorato dalla procedura penale ebraica). E' interessato alla dottrina ed ai seguaci di Gesù, e vuole capire se esista una setta con finalità eversive, cosa che sarebbe assai utile nella successiva fase processuale presso il tribunale romano, innanzi a Pilato. ${ }^{40} \mathrm{Ma}$ l'interrogatorio non approda ad alcun risultato e di conseguenza Gesù viene mandato

37 Marco, 14, 43-44.

38 J. Blinzler, Il processo..., op. cit., p. 77; M. Miglietta, I. N. R .I...op. cit., p. 34.

39 J. Blinzler, Il processo..., op.cit., pp. 83-84; Carlo Nardi, Il processo di Gesù Re dei Giudei, Bari, 1966, p. 89.

40 B. Fabbrini, convegno Le accuse e le prove: "E' da ritenere che il colloquio con Anna, insignificante dal punto di vista giuridico, sia servito a indagare meglio sulla duplice natura dei reati da ascrivergli, cioè blasfemia e sedizione". 
da Caifa per l'udienza formale. E' verosimile che Anna e Caifa abitino due ali dello stesso palazzo, visto che la traduzione di Gesù nell'aula del dibattimento, davanti a Caifa ed al Sinedrio, richiede pochi minuti. E' improbabile che tutti i sinedriti siano presenti: la totalità dei 71 componenti è infatti richiesta solo in casi di idolatria ed in cause riguardanti i Sommi Sacerdoti; in materia penale sono richiesti 23 membri.

A differenza dell'interrogatorio di Anna, riportato dal solo Giovanni, dell'udienza tenuta davanti al Sinedrio sono solo i sinottici a parlare. La ragione di questa mancanza sta forse nella cronologia dei vangeli, che vede quello di Giovanni essere l'ultimo, quando ormai gli altri hanno già ampiamente parlato del dibattimento.

Come visto, il Sinedrio è composto da un gruppo dei sacerdoti, che Blinzler definisce crudamente «ierocrati assetati di potere, laicizzati e senza coscienza». ${ }^{41} \mathrm{Il}$ secondo gruppo è quello degli anziani del popolo, rappresentanti delle famiglie più ricche ed influenti di Gerusalemme. Tra essi è Giuseppe d'Arimatea, che si incaricherà della deposizione e sepoltura di Gesù nella tomba nuova di sua proprietà, nei pressi del Golgota. Al terzo gruppo appartengono gli scribi, in parte farisei, che detengono il potere amministrativo e giudiziario. Sono accaniti oppositori del predicatore nazareno a causa del suo ascendente sul popolo, anche se tra loro c'è qualcuno meno estremo: sicuramente Nicodemo, che ritroveremo al momento della sepoltura di Gesù col suo sontuoso dono di profumi ed aromi, degno di una sepoltura regale. ${ }^{42}$

La procedura processuale ebraica non è basata sul ruolo preminente dell' accusa: qui sono i testimoni che, favorevoli o contrari all'imputato, determinano lo svolgimento del procedimento. Prima si escutono i testi favorevoli all'imputato, successivamente quelli contrari. Anche una sola testimonianza favorevole e credibile deter-

${ }^{41}$ J.Blinzler, Il processo..., op. cit., p. 155.

42 Giovanni, 19, 38-40 (Giovanni parla di 100 libbre tra mirra ed aloe, corrispondenti a circa $32-33 \mathrm{Kg}$ !!). 
mina la non incriminazione dell'imputato, mentre per incriminarlo è richiesta la testimonianza concorde di almeno due testi contrari. ${ }^{43}$ Ma di testi a favore non c'è traccia; infatti i vangeli non ne parlano e i discepoli si sono dileguati. I testimoni d'accusa, invece, malgrado l'ora sono già pronti: è verosimile che siano stati avvisati per tempo, visto che l'arresto di Gesù era stato programmato la sera precedente.

Vengono ascoltati alcuni testi separatamente, ma le loro testimonianze sono discordi e quindi inutilizzabili. Qualche studioso parla di testi preparati e comperati, ${ }^{44}$ per Blinzler invece si tratta di un possibile errore di regia; altri invece sostengono che la discordanza tra $\mathrm{i}$ testi è indice di serietà della procedura. ${ }^{45}$ Mancando testimonianze valide, è Caifa ad interrogare l'imputato: se Gesù riconosce come vera almeno una delle accuse le discordanze vengono cancellate, e si costruisce la base per un’imputazione. Ma Gesù tace, ed il dibattimento giunge ad un punto morto. Ciò induce Caifa ad una domanda diretta, quella riguardante la presunta messianicità di Gesù, per capire se questi possa essere accusato di blasfemia.

Parte della dottrina ritiene che per la legge ebraica nessuno possa essere condannato sulla sola sua confessione, ${ }^{46}$ ma Miglietta lucidamente rileva che qui non si tratta di confessione o di testimonianza, perché Gesù si rende reo di bestemmia proprio davanti al Sinedrio: ${ }^{47}$ Infatti, alla precisa domanda se fosse il Cristo, Gesù risponde "io lo sono". ${ }^{48}$ Gesù si dichiara dunque, davanti alle più alte

43 Deuteronomio, "Un condannato non sia messo a morte se non sulla deposizione di due o tre testimoni $[\ldots]$ ".

44 R. Gutzwiller, Gesù il Messia, Monaco, 1949, p. 319.

45 B. Fabbrini, Le accuse..., op. cit. "[...]il fatto che le accuse dei testimoni, che potevano portare al reato di blasfemia non siano accettate, è prova evidente della serietà di questo processo sinedrile".

46 Tra i vari: J. Imbert, Il processo a Gesù, Brescia, 1980, p. 64.

47 M. Miglietta, I. N. R. I., op. cit., p. 37.

48 Marco, 14, 61-62; similmente Luca, 22, 67-70 e Matteo, 26, 63-64. 
autorità del suo popolo, uguale a Dio: si tratta di una bestemmia pronunciata in pubblico, e si giustifica il gesto di orrore di Caifa, che si straccia le vesti dichiarandolo meritevole di morte. Si ricordi che meritevole di morte non significa condannato, ma deferibile al tribunale romano di Pilato, poiché il Sinedrio è privo della potestà di emettere sentenze capitali.

Secondo Marco e Matteo appena giorno i sinedriti si riuniscono in consiglio e, dopo aver fatto legare Gesù, lo conducono da Pilato. Questo tenere consiglio indica come l'accusa di colpevolezza di Gesù sia assunta con decisione collettiva, per arrivare da Pilato con maggior forza sostanziale.

\section{v. Il giudizio del magistrato romano Ponzio Pilato}

Esaurita la procedura accusatoria giudaica, che ha portato alla sola accusa di blasfemia per essersi Gesù dichiarato uguale a Dio, Gesù viene condotto da Ponzio Pilato. E' lo stesso Pilato che si fa incontro al gruppo dei sinedriti, che non entrano nella sua residenza di Gerusalemme per non cadere nell'impurità che proibirebbe loro di consumare la Pasqua. I vangeli chiamano Pretorio il luogo del processo romano a Gesù, cosa che non può che indicare la residenza ufficiale del governatore. ${ }^{49}$

E' nel momento della consegna a Pilato che si colloca, ad opera del Sinedrio, la trasformazione del capo d'accusa: da bestemmia, alla cui persecuzione Pilato avrebbe certo posto rifiuto, a lesa maestà. E' palese l'astuzia di portare sul piano politico l'accusa, sostenendo essersi Gesù fatto re dei Giudei e di aver commesso altri delitti, quali la sedizione e l'incitamento alla rivolta fiscale. Imbert, però, osserva come basti leggere i vangeli per accorgersi che «le accuse fanno acqua da tutte le parti». ${ }^{50}$ La riprova è fornita dai vari

49 J. Blinzler, Il processo..., op. cit., p. 233.

50 Idem, p.99. 
tentativi di Pilato di rilasciare Gesù, cioè i ripetuti interrogatori, il tentativo di scambio con Barabba, l'invio ad Erode Antipa, il lavaggio delle mani. ${ }^{51}$

C'è da chiedersi perché i sinedriti abbiano condotto un procedimento contro Gesù, ben sapendo che un' incriminazione per motivi religiosi non avrebbe avuto alcun valore: ecco l'astuzia ed il realismo di portare i loro interessi religiosi sul piano politico, attraverso l'accusa essersi attribuito il titolo di re. Si tratta del reato di lesa maestà, quel crimen maiestatis che metterebbe in pericolo la sicurezza della provincia romana, ledendo il prestigio e la maestà dell'imperatore, cose che Roma persegue e punisce con la morte.

Pilato, dopo aver tentato di lasciare ai giudei il compito di processare Gesù secondo le loro leggi, pensando trattarsi di una delle solite questioni religiose locali, e dopo aver ricevuta la risposta "a noi non è consentito di dare morte ad alcuno", 52 inizia ad interrogare Gesù. La narrazione dei vangeli è coerente nei sinottici, pur con una maggior precisione di Luca riguardo alle accuse: alla precisa domanda di Pilato "sei tu il re dei giudei?", Gesù risponde lapidario "tu lo dici". Le altre accuse, malgrado la reiterata insistenza dei sacerdoti, si rivelano inconsistenti e Pilato le lascia cadere, di fronte al mutismo di Gesù che lo lascia meravigliato e che lo induce ad affermare di non trovare in lui alcuna colpa. ${ }^{53}$ Giovanni è l'unico a parlare dell'andirivieni di Pilato tra il Pretorio ed i Giudei, e lui pure cita la risposta di Gesù "tu l'hai detto, io sono re" alla domanda di Pilato.

Pilato è dunque tutt'altro che convinto delle accuse dei sinedriti e, quando viene a sapere che su Gesù avrebbe giurisdizione Erode Antipa, re della Galilea

51 M. Miglietta, I.N.R.I., op. cit., p. 44.

52 Giovanni, 18, 31.

53 Luca, 23, 1-5; Giovanni, 18, 33-38. 
in quei giorni a Gerusalemme, glielo invia. ${ }^{54} \mathrm{Ma}$ qual è lo scopo di Pilato? Non è sostenibile che si tratti di motivi di competenza, perché Gesù è giudeo, anche se a lungo ha vissuto in Galilea; soprattutto non è pensabile che Pilato, che detiene il ius gladii, sia tanto autolesionista da consegnare ad Erode un preteso ribelle giudeo, punibile con la pena di morte per reati commessi in Giudea. E' invece il tentativo di Pilato, convinto dell'innocenza di Gesù, di avere un'autorevole opinione di conforto da far valere contro il Sinedrio e, con un atto di real politik, migliorare i suoi pessimi rapporti con Erode che, ricordiamo, era "persona gratissima» presso Tiberio. ${ }^{55}$

Ma anche alle domande di Erode si contrappone l'ostinato mutismo di Gesù, così che non ci sia altro da fare che rimandarlo a Pilato dopo averlo schernito e dileggiato, ma senza aver trovato in lui alcuna colpa. Ricondottogli Gesù, che Pilato annuncia di voler sottoporre a flagellazione per poi liberarlo perché non meritevole di condanna a morte, invece di procedere in questo senso sceglie di riprendere il dibattimento, con ciò implicitamente segnando la sorte di Gesù.

Gli evangelisti parlano poi di una tradizione secondo la quale il governatore era solito per Pasqua liberare un prigioniero a richiesta della folla. Pilato intravvede in questa concessione la possibilità di liberare Gesù, confidando che, posta innanzi all'alternativa Gesù-Barabba (ribelle accusato di omicidio), la folla scelga Gesù: ma così non è, perché l'opera di sobillazione dei sacerdoti convince la folla a richiedere la libertà di Barabba. Si tratta di un palese errore di valutazione, aggravato dal mettersi a discutere del destino di Gesù con una folla ormai schierata. ${ }^{56}$

Ma il Privilegium Paschale esiste od è fantasia degli evangelisti? La dottrina si è divisa: una parte giudica inattendibili i vangeli su tale

54 Luca, 23, 6-12.

55 G. Flavio, Antichità..., op. cit. 18,2,3; G. Ricciotti, Il cantiere di Himran, Milano, 1950, p. 172.

56 J. Blinzler, Il processo..., op. cit., pp. 280-281. 
argomento; ${ }^{57}$ la parte maggioritaria l'ammette, pur spiegandolo variamente. Secondo il pur radicale demitizzatore Martin Dibelius, «Anche se non sappiamo nulla di tale usanza, non vi è alcun motivo di dubitare di questa scena». ${ }^{58}$ Per Mommsen è un diritto del giudice di archiviare il processo, ${ }^{59}$ Henry Regnault e Johannes Merkel ritengono che si tratti di un potere di amnistia concesso dall'imperatore al governatore. ${ }^{60}$ Un papiro ritrovato nel 1906 parla di un procedimento contro tale Fibione dinnanzi a Settimio Vegeto: il caso è analogo a quello di Barabba ed anche in questo caso viene liberato un malfattore a richiesta del popolo. ${ }^{61}$ Da parte ebraica, un passo del Pesachim VIII 6-a dà prova dell'usanza di liberare uno o più detenuti per Pasqua. Siamo dunque di fronte ad una tradizione attestata sia nel mondo romano sia in quello ebraico.

Il diritto romano prevede due forme di amnistia: l'abolitio, rilascio di un prigioniero non ancora condannato, e l'indulgentia, cioè la grazia concessa ad un condannato. Quella che Pilato pensa di applicare a Gesù, e che finirà invece coll'accordare a Barabba, è chiaramente l'abolitio: infatti Gesù non è ancora stato condannato e neppure lo è Barabba, descritto semplicemente come prigioniero.

L'atteggiamento di Pilato in tutta la vicenda ha originato svariate interpretazioni riguardo alle sue convinzioni personali ed al suo comportamento sul piano giuridico-processuale. Appare evidente, circa le sue convinzioni, come non veda in Gesù un rivoluzionario od un eversore: è probabile che non ne abbia mai sentito parlare e

57 P. Van Paassen Why Jesus Died, 1949, p.171; C. Guignebert, Iesus, 1947, pp. 573-575; J.Isaac, Iesus et Israel, 1948, p.485; M. Goguel, Le procès de Jésus, 1949, p. 382.

58 M. Dibelius, Jesus, 1939, p. 116.

59 T. Mommsen, Romisches Strafrecht, Lipsia, 1899, p. 453.

60 H. Regnault, Le procès de Jésus Christ, Parigi, 1909, p. 133; J. Merkel, ZNW 6, 1905, p. 303.

61 Pap. Flor., 61, 59 e segg. "Avresti meritato la frusta [...]ma io ti abbandonerò alla folla, così ti tratterò umanamente". 
che lo consideri uno dei tanti predicatori di una religione che sfugge alla sua comprensione. Per questo sembra determinato a salvare Gesù da una morte frutto di accuse che per lui hanno scarso significato. Se si aggiunge il fastidio e disprezzo che nutre per il Sinedrio ed i suoi rappresentanti, si può comprendere perché una persona dal carattere duro quale è Pilato, determinato e spietato quando si tratta di difendere gli interessi di Roma, cerchi di rilasciare Gesù. Quindi non compassione od umanità, ma il tentativo di non lasciarsi coinvolgere in fatti che non riguardano il controllo della provincia ed il suo potere e che, soprattutto, non hanno interesse processuale.

Dall'istruttoria emerge però che Gesù si è professato Messia, Figlio di Dio e re dei Giudei, cosa questa che sul piano politico equivale al titolo di re d'Israele. Per i romani ciò configura un reato, perché minaccia la sicurezza del popolo romano e la maestà dell'imperatore. ${ }^{62} \mathrm{Si}$ comprende quindi la circospezione di Pilato negli interrogatori, che si concentrano sul reato di lesa maestà solo sotto la spinta dei ricatti e delle minacce giudee. Infatti è dopo l'ultimo confronto con Gesù e l'estremo tentativo di liberarlo, frustrato dal grido minaccioso "se lo liberi non sei amico di Cesare, infatti chi si fa re non è amico di Cesare", che Pilato cede. ${ }^{63}$ Si tratta di una minaccia che Pilato non può ignorare o sottovalutare, per il rischio reale di una denuncia a Roma da parte del Sinedrio.

Terminata la fase del giudizio, Pilato emette formalmente la condanna: Gesù deve essere crocifisso.

Lo svolgimento del processo è tipicamente romano, con l'emissione di una sentenza di condanna da parte del magistrato romano, che siede in tribunale prendendo posto sulla sella curulis. ${ }^{64}$ Tutto questo in antitesi con l'ipotesi che si tratti della semplice delibazione di una sentenza del Sinedrio. Abbiamo del resto almeno quattro con-

\footnotetext{
62 M. Miglietta, I. N. R. I., op. cit., p. 46.

63 Giovanni, 19, 12.

64 T. Mommsen, Le droit public..., op.cit., p. 298.
} 
ferme che ci sia un'effettiva sentenza di condanna e la conseguente esecuzione: la consegna di Gesù ai soldati romani; la crocifissione tipicamente romana e non la lapidazione giudaica, peraltro non più possibile; l'apposizione del titulus crucis ed il rifiuto di modificarlo malgrado le pressioni dei sinedriti; la richiesta del corpo di Gesù fatta da Giuseppe d'Arimatea a Pilato, che la concede.

Si è trattato allora di un duplice processo, quello sinedrile e quello romano, oppure di uno soltanto? Un processo religioso per blasfemia, senza poter condannare Gesù ed eseguire la pena, non avrebbe avuto senso; i sinedriti, anzi, al momento di consegnarlo a Pilato hanno modificato le accuse in sedizione, ribellione fiscale ed affectatio regni. Ancor meno credibile è che sia il Sinedrio a condannare Gesù e che Pilato si limiti a delibare la sentenza: è un'ipotesi mai adombrata dai vangeli e contraria allo svolgimento processuale romano. ${ }^{65} \mathrm{Il}$ processo è dunque uno solo, diviso in due fasi: prima l'istruttoria sinedrile, nel corso della quale Gesù si rende reo di blasfemia e viene deferito al tribunale romano, ma con cambio del capo d'accusa; poi il giudizio di Pilato e la sentenza di condanna a morte per laesa maiestas.

Resta da chiedersi se Pilato non abbia agito con leggerezza: Miglietta giunge alla conclusione che «Pilato giudicò con la severità imposta da Tiberio, e dunque in conformità con la giurisprudenza dell'epoca». ${ }^{66}$ Nardi, che pure condivide l'esistenza di un unico processo in due fasi, è invece critico sul ruolo di Pilato, ed afferma: «Il giudice che con piena e libera coscienza, o per coartazione fisica o morale, condanna invece di assolvere un innocente è in ambedue i casi colpevole contro il dovere del suo mandato, e nulla conta che abbia seguito o no la procedura. Quel che conta è la sentenza, che

65 G. Nardi, intervento al congresso "Storia, antichità greca e romana", Univ. di Genova, p. 43.

66 M. Miglietta, I. N. R. I., op. cit., p. 53. 
non è più espressione del suo giudizio, ma della prepotente volontà dell'accusatore». ${ }^{67}$

Sicuramente sono entrati in gioco interessi politici, ed il timore di compromettere il prestigio e la stabilità della carica per un fatto per lui di scarso interesse ma complicato dal rischio di possibili rivolte o ricorsi a Roma, cose esiziali per la carriera e la sua stessa persona. Certo processo e condanna sono improntati a correttezza formale, ma altrettanto certamente mostrano un comportamento moralmente discutibile: è percepibile la sua dimostrazione di viltà al sentirsi accusare di non essere amico di Cesare ed il piegarsi agli accusatori. ${ }^{68}$

VI. Esecuzione deLla CondanNa

La parte iniziale dell'esecuzione della condanna alla croce è la flagellazione. Nel processo criminale romano di regola la flagellazione è una pena collaterale che precede la crocifissione, anche se a volte accade che il disgraziato muoia direttamente sotto i colpi del flagello. Mentre Luca accenna solo all'intenzione di Pilato di far flagellare Gesù, Marco e Matteo ne parlano espressamente. A differenza dei giudei, che applicano la flagellazione col limite massimo di quaranta colpi, ${ }^{69}$ tra i romani è ad libitum e lasciata all'arbitrio dei carnefici. Nelle provincie l'esecuzione è affidata ai militari, che utilizzano il flagello per sudditi provinciali e schiavi. Viene utilizzato il flagrum taxillatum, costituito da un'impugnatura alla quale sono collegate corde o strisce di cuoio, che terminano con pezzetti d'osso o piccoli

67 C. Nardi, Storia, antichità..., op. cit., pp. 45-46.

68 Dante, La divina commedia, Inf. I, 3, 58-60. "[...] vidi e conobbi l'ombra di colui che fece per viltà il gran rifiuto". Dante non nomina il personaggio che cita, ma la critica, a partire dal secolo scorso (per tutti Giovanni Pascoli, Nicola Griniti, Natalino Sapegno), è sempre più indirizzata ad identificarlo con Pilato.

69 Deuteronomio, 25, 2-3; Paolo, II lettera ai Corinzi, 2, 24 "per cinque volte ho ricevuto quaranta colpi meno uno". 
bilancieri di piombo. ${ }^{70}$ L'effetto di questo strumento è devastante, e le narrazioni dell'antichità evidenziano un quadro orribile. ${ }^{71}$

Nel caso di Gesù è credibile che Pilato, dopo aver impartito ai suoi uomini l'ordine di flagellare in luogo pubblico e crocifiggere il condannato, si sia ritirato lasciando loro il compito di provvedere. E' pure credibile che i soldati provvedano per dileggio all' "incoronazione" di Gesù con un casco di spini: si tratta di un gesto di irrisione per niente inverosimile da parte di un gruppo di rozzi ed incattiviti soldati, che hanno in mano un condannato che non ha più alcun diritto e che non li muove a compassione. ${ }^{72}$ Non conosciamo con certezza le modalità della flagellazione di Gesù né la sua entità, anche se vedremo nei capitoli seguenti, parlando della Sindone, che le tracce impresse sul sudario mostrano almeno 120 colpi. Senza dubbio i traumi prodotti dalla flagellazione condizionano gravemente il fisico di Gesù, già provato dalle vicende processuali e dalle percosse ricevute: si può ragionevolmente considerare questa punizione, seguita dalla via crucis verso il Golgota, come una delle principali cause della sua rapida agonia in croce. Infatti, dalla condanna alla morte, tutto si compie tra l'ora sesta e la nona (tra mezzogiorno e le quindici), meno di tre ore, cosa che provoca la meraviglia di Pilato quando Giuseppe d'Arimatea gli domanda il corpo di Gesù per la sepoltura: infatti la morte in croce è lunga e drammatica, e l'agonia può durare anche uno o due giorni.

Gesù, dopo la flagellazione, viene subito inviato verso il luogo in cui deve essere crocifisso. Non porre tempo tra condanna ed esecuzione è cosa normale; gli antichi, ed i romani non fanno eccezione, non conoscono le pene detentive: la prigione serve solo a trattenere l'imputato che attende il giudizio. Il tragitto che Gesù

70 Presso il Museo Nazionale delle Terme di Roma è conservato un flagrum taxillatum che, per gli studiosi, è simile a quello usato nei confronti di Gesù.

71 G. Flavio, La guerra..., op.cit., 6, 5, 3; Cicerone, In Verrem, 2, 4, 39, 85; idem, 5, 11,1; Prudenzio, citaz. di M. Centini.

72 G. Ricciotti, Vita di Gesù, op.cit., p. 177. 
deve compiere con il patibulum sulle spalle, che può arrivare a pesare anche cinquanta $\mathrm{Kg}$., ha funzione di debilitare ancor più un fisico già provato dalla flagellazione, ma anche di ammonimento per chi assiste. Di patibulum si tratta e non della croce tramandata dall'iconografia: infatti è quasi impossibile per un condannato portare una croce di oltre tre metri, del peso di molte decine di Kg. Che il condannato alla croce porti il suo patibulum lo confermano con precisione Plauto ${ }^{73}$ ed Artemidoro. ${ }^{74}$

La crocifissione, pur se attribuita a greci, cartaginesi e persiani, è divenuta nei secoli una pena tipicamente romana. Ne parlano abbondantemente molti autori romani, descrivendola come «pena crudele, miseranda e spaventosa». ${ }^{75}$ Da Cesare sappiamo che lui stesso, in Spagna, fece crocifiggere tre schiavi. ${ }^{76}$ Nelle provincie romane questa pena diventa uno dei principali mezzi per il mantenimento dell'ordine pubblico, e la turbolenta Giudea annovera numerose esecuzioni di questo genere. Basti dire che Varo (lo stesso di Teutoburgo) mandò in croce oltre duemila giudei e che, durante l'assedio di Gerusalemme, vennero a mancare il legno per le croci e lo spazio per innalzarle. ${ }^{77}$

La croce romana è composta da due legni separati, lo stipes ed il patibulum. Lo stipes è la parte verticale della croce, un tronco od un palo già infisso in terra. Può essere poco più alto di un uomo e si tratta della crux humilis destinata all'uso normale. Nel caso di una condanna alla quale si voglia dare maggior risalto lo stipes è più alto, anche oltre i tre metri: si tratta della crux sublimis, la stessa utilizzata nel caso di Gesù. Il patibulum è la trave separata che viene

73 Plauto, Carbonaria, 2 "Che porti il patibolo per la città e poi sia affisso alla croce".

74 Artemidoro, Oneir., 2, 56 "chi deve essere affisso alla croce, prima la porti".

75 Cicerone, Pro Rabirio, 5,16; idem, In Verrem, 2, 5,64; G.Flavio, La guerra..., op.cit, 7, 6, 4; Plauto, Miles Gloriosus, 372.

76 Cesare, De Bello Hispanico, 20,5.

77 G. Flavio, La guerra..., op.cit., 5,11, 1. 
unita allo stipes sul luogo della crocifissione; è caricato sulle spalle del condannato e legato alle braccia divaricate perché non scivoli e, soprattutto, per evitare che questi se ne liberi e tenti di fuggire. La forma della croce differisce a seconda che il patibulum venga fissato in cima allo stipes, formando una sorta di T (crux commissa), oppure inserito in un incavo dello stipes (crux immissa): è la croce a quattro braccia che conosciamo, sulla cui parte terminale viene apposto il "titulus crucis".

Il titulus crucis è generalmente una tavoletta a fondo bianco con iscrizioni in rosso, che riporta le motivazioni della sentenza di condanna. Nel caso di Gesù l'iscrizione, che ci è nota con l'acronimo latino I.N.R.I., è trilingue e dice «Gesù Nazareno Re dei Giudei»: è una frase che i sacerdoti vorrebbero far modificare, cosa che ottiene lo sprezzante rifiuto quod scripsi scripsi di Pilato. ${ }^{78}$ Non si tratta solo di un sussulto di autorità e coraggio, quelli mancati nel pur formalmente corretto processo: rappresentando infatti il titulus la sentenza di condanna emessa, questa non poteva più essere modificata. Su questa tavoletta, una parte della quale si ritiene essere quella conservata nella Basilica romana di Santa Croce in Gerusalemme, esistono diversi studi ed opinioni. Uno studio recente, molto ben argomentato e documentato, ripercorre la storia del suo ritrovamento da parte di Elena, madre di Costantino, ed è opera del giornalista, storico ed antropologo M. Hesemann. ${ }^{79}$ Il reperto evidenzia qualche difficoltà da parte dell'esecutore, probabilmente ebreo, che scrive i testi greco e latino da destra a sinistra, come d'uso per l'ebraico; inoltre qui il testo greco precede quello latino, a differenza di quanto narra Giovanni. ${ }^{80}$ In ogni caso, che si accetti la descrizione di Giovanni o quella espressa nel titulus conservato a Roma, la condanna per il reato di lesa maestà appare incontrovertibile.

78 Giovanni, 19, 21-22.

79 M. Hesemann, Titulus Crucis, Torino, 2000.

80 Idem, pp. 347-348. 
Ci si è ripetutamente chiesti se la crocifissione sia avvenuta utilizzando i chiodi oppure la corda: la questione è attendibilmente risolta con l'accettazione della tesi dei chiodi. ${ }^{81}$ Per secoli l'iconografia ha mostrato come al patibulum fossero inchiodate le mani, precisamente i palmi. Vedremo però come una serie di esperimenti dello scorso secolo di un medico francese, nati dall'esame della Sindone, mostrino come i chiodi fossero piantati nei polsi: infatti i palmi trafitti non reggono il peso di un corpo abbandonato, mentre le ossa del polso bloccano il chiodo e sostengono il corpo. ${ }^{82}$ I carnefici romani, pur non avendo studiato anatomia, avevano un'esperienza tale da ben conoscere dove infiggere i chiodi.

Quanto ai piedi, i sistemi di crocifissione prevedevano due possibilità: nel primo caso sullo stipes veniva fissato una sorta di sgabello (sedilis) sul quale i condannati sedevano a gambe divaricate, ${ }^{83}$ e ciò portava ad inchiodare i piedi separatamente, essendo difficoltoso sovrapporli. Nel secondo caso non esistevano sgabelli o simili, ed al condannato un unico chiodo trafiggeva i piedi sovrapposti. L'esistenza di un sedilis presuppone il prolungamento dell'agonia, potendo il condannato appoggiarsi; nell'altro caso è una possibilità preclusa, col crocifisso che deve far forza sui piedi inchiodati per sollevarsi. Per Gesù e gli altri due condannati non esiste alcun sostegno, e la prova inoppugnabile consiste nell'effettuazione del crurifragium ai condannati, richiesto a Pilato dai sacerdoti nell'intento di accelerarne la morte, affinché muoiano e siano inumati prima della Pasqua imminente. ${ }^{84}$ Se avessero avuto il sedilis, lo spezzare le gambe avrebbe certo causato sofferenze aggiuntive, ma non avrebbe garantita la celere morte richiesta dalla particolare occasione.

81 Tra i tanti: Plauto, Mostellaria, 359-360; Seneca, Dialoghi, 7, 19,3; E. Cantarella, I supplizi capitali, Milano, 2005, pp. 211-213; C.Augias, M. Pesce, Inchiesta..., op.cit., p. 164; Paolo, Lett. ai Colossesi, 2, 13-14.

82 P. Barbet, La Passion de N.S.fésus Christ selon le chirurgien, Issoudun, 1950.

83 G. Flavio, La guerra..., op.cit., 2,14,9.

84 Giovanni, 19, 31-32. 
Quando i soldati, dopo aver effettuato il crurifragium agli sventurati compagni di Gesù, si volgono a Lui, si rendono conto che è già morto e non lo colpiscono: uno di essi, però, per sicurezza infligge un colpo di lancia al costato, dal quale sgorgano sangue ed acqua (siero). ${ }^{85}$

Riguardo alla fine di Gesù, la narrazione degli evangelisti è scarna su quel che accade sulla croce. Ciascuno, secondo i diversi ricordi e la personale inclinazione, dà spazio ai vari personaggi presenti all' agonia e morte di Gesù, ed alle sue parole in croce. In Marco e Matteo nessuna parola, tranne la disperata invocazione finale "Eloi, Eloi, Lamà Sabactani?" (Dio mio, Dio mio, perché mi hai abbandonato?), seguito dall'inarticolato forte grido che precede la subitanea morte. ${ }^{86}$

Così Gesù, accusato dai sinedriti di essersi proclamato re dei Giudei e di volersi fare Cesare, condannato da Pilato per il crimine di lesa maestà, chiude poco regalmente la sua vita terrena.

Appurato e comunemente accettato che un insieme di ragioni hanno portato alla morte Gesù, stress, debilitazione, disidratazione, episodi settici, difficoltà respiratorie, insufficienza cardiaca, ecc., i patologi hanno indagato sulla causa primaria della morte, soprattutto legandola alle difficoltà respiratorie e alla conseguente morte per asfissia. ${ }^{87}$ In tempi più recenti non il soffocamento ma un'ischemia cardiaca è indiziata di essere causa della repentina morte, per sovraffaticamento del cuore causato anche dal sangue denso per la disidratazione. Ciò concorda col grido finale di Gesù, impensabile in caso di soffocamento, e con l'effusione di sangue e siero al colpo di lancia del soldato. ${ }^{88}$

85 Idem, 19, 33,34.

86 Marco, 15,34; Matteo, 27,46.

87 R. W. Hynek, Golgota, Praga, 1936; M.G.Siliato, Sindone, Casale Monferrato, 1997, pp. 308-310.

88 Tra gli altri, G.Ricci, La Sindone Santa, Roma, 1976, pp. 488-489; P. L. Baima Bollone, Gli ultimi giorni di Gesù, Firenze, 2003, pp. 56-70. 
Nell'usanza romana il cadavere del crocifisso non viene sepolto, ma resta appeso per più giorni quale ammonimento e deterrente. Per i giudei questo è inconcepibile, perché l'appeso è considerato una maledizione del Signore e deve essere sepolto il giorno stesso della morte. A maggior ragione se si tiene conto dell'imminenza della Pasqua, che verrebbe impedita dalla contaminazione della città che l'esposizione dei cadaveri provocherebbe. Ecco il motivo della pressante richiesta a Pilato di far rompere le gambe ai crocifissi: la conseguente rapida morte consentirebbe la loro sepoltura prima del tramonto. Non conosciamo chi si occupi degli altri crocifissi, presumibilmente si tratta di incaricati del Sinedrio. Di Gesù sappiamo dai vangeli che se ne prende carico Giuseppe d'Arimatea, ricco membro del Sinedrio, uomo dabbene e suo discepolo occulto, che si reca da Pilato per richiederne il corpo. Pilato, avuto conferma della morte di Gesù dal centurione, asseconda la richiesta e ne dispone la consegna. ${ }^{89} \mathrm{La}$ consegna ai familiari di un giustiziato è attestata dall'autobiografia di Augusto, che si vanta di essersi sempre attenuto alla prassi di non rifiutare la consegna del corpo del condannato ai parenti che lo richiedevano. ${ }^{90} \mathrm{E}$ ' logico supporre che anche Tiberio si sia attenuto a questa prassi, nella cui scia si deve analizzare il permesso concesso da Pilato: ${ }^{1}$ Giuseppe, pur non parente del giustiziato, si presenta a Pilato con credenziali che lo convincono ad assecondare la richiesta. Che poi Gesù venga inumato in una tomba privata e non in quella comune a Pilato non importa, trattandosi di norme mortuarie ebraiche che non lo interessano. Per i giustiziati ebrei è previsto un luogo pubblico di sepoltura e solo dopo un

89 B. Fabbrini, La deposizione di Gesù nel sepolcro, SDHI LVI, pp. 158-159; idem, p.178 "I vangeli mostrano di essere una fonte importantissima per gli studiosi di diritto romano. Sono una delle pochissime fonti del I sec. d.C. ad attestare un processo penale provinciale[...] compresa la fase della deposizione dalla croce e della sepoltura".

90 Svetonio, Augusto, 13.

91 T. Mayer Maly, Deposizione e sepoltura, SDHI LVI, p.95. 
anno, quando le salme saranno decomposte, la colpa sarà considerata estinta e le ossa potranno essere riunite nell'ossario di famiglia senza più rischio di contaminazione. Gesù viene invece deposto in una tomba nuova scavata nella roccia, nella quale nessuno è stato mai sepolto, ${ }^{92}$ e ciò spiega perché i sacerdoti non si oppongano: anche se situazione privilegiata, non c'è rischio di contaminazione o disonore perché nessun altro vi è deposto.

I tre sinottici sono sintetici ed assai simili nel descrivere la deposizione e sepoltura di Gesù: da loro sappiamo che Giuseppe compra un telo, depone il corpo di Gesù, lo avvolge nel telo e lo pone nel suo sepolcro nuovo scavato nella roccia. Giovanni fa anche intervenire Nicodemo, ricco discepolo occulto di Gesù, che compera 100 libbre di aromi ed aiuta Giuseppe a deporre ed avvolgere Gesù. I vangeli non parlano di una lavanda del corpo, ed è verosimile che questa non sia stata fatta. La sepoltura di Gesù avviene infatti secondo l'uso ebraico, per il quale sono quattro le categorie di persone che non ricevono la purificazione rituale: vittime di morte violenta, giustiziati per crimini di natura religiosa, proscritti dalla comunità ebraica, uccisi da non giudei. Per i giudei Gesù è incluso in tutte queste categorie. Nella narrazione di Luca e Marco, le donne che la domenica mattina si recano alla tomba vanno con profumi ed unguenti, ma non per lavarlo. ${ }^{93}$

\section{LE FONTI}

I Vangeli sono, insieme con gli Atti degli apostoli e le lettere di Pietro e Paolo, le fonti dirette principali se non uniche. Di dubbia attendibilità sono i vangeli apocrifi, che lasciano trasparire un chiaro intento apologetico ed una narrazione che spesso sconfina nella fantasia descrittiva: non a caso la chiesa li ha espunti fin dall'inizio dai suoi documenti

\footnotetext{
92 Luca, 23, 53; Giovanni, 19, 41.

93 Luca, 24, 1; Marco, 16, 1.
} 
ufficiali, anche a costo di lacerazioni nel suo interno. Quanto alle fonti non cristiane, da esse possiamo trarre notizie, precisazioni, conferme; nessuna però offre testimonianze dirette della vicenda processuale di Gesù. Giuseppe Flavio, Tacito, Svetonio, Ulpiano, al pari di altri coevi, sono certo essenziali, ma "di cornice", confermando od attestando quanto narrato dai vangeli.

Ancora in tempi recenti si è manifestato scetticismo od avversione sull'autenticità ed attendibilità dei vangeli, perché sarebbero frutto di stesure tardive, quando non costruiti ad arte. Si è parlato di stesure che vanno dal 70 per Marco al 100 d.C. per Giovanni, affermando come il trascorrere degli anni non sia testimonianza di genuinità o, peggio, che si tratti di costruzioni artificiali di non meglio precisate comunità cristiane primitive. Con questo discutibile modo di ragionare che credibilità possono avere allora gli Annales di Tacito quando parla di Tiberio, che ha iniziato il suo principato cento anni prima? E quanta Svetonio, che nelle sue Vite dei Cesari narra dei cesari a partire da Giulio Cesare, cioè due secoli prima? Eppure queste sono state accettate senza obiezioni nei secoli da storici e studiosi. Un altro particolare troppo facilmente dimenticato è che venti secoli or sono non esistevano le odierne possibilità di scrittura, registrazione di eventi ed immagini; gran parte della popolazione era analfabeta, e chi sapeva scrivere non era certo facilitato dall'uso di papiri e pergamene. La necessità era dunque quella della memorizzazione e trasmissione orale, cose per secoli praticate in occidente ed ancor oggi nel mondo islamico.

Ma se queste pur evidenti argomentazione non bastassero, ecco intervenire il caso e l'intuizione a fornire decisiva attendibilità ai vangeli: si tratta dalla scoperta, nel secolo scorso, dei rotoli di Kumran e del papiro Magdalen, che retrodatano in maniera decisiva i vangeli di Marco e Matteo, attestandoli a pochissimi decenni, ed ancor meno nel caso di Marco, dalla morte di Gesù.

Kumran identifica un isolato insediamento esseno sopra il mar Morto, distrutto nel 68 d.C. dai romani. I ritrovamenti in questa de- 
solata località iniziano nel 1945, quando un pastore beduino scopre la prima anfora in una delle tante grotte. Gli archeologi ne vengono a conoscenza nel 1947, quando trovano in vendita antichissimi manoscritti presso antiquari di Gerusalemme. Le grotte vengono setacciate, e nel 1955 è il turno della grotta identificata come 7Q: il risultato è a prima vista deludente, soltanto diciotto frammenti di papiro in greco. E' chiaro che la grotta è già stata ripulita in precedenza. Da rilevare che in nessuna altra grotta vengono ritrovati testi in greco. Il più grande frammento viene identificato con la sigla 7Q5, con scritte su cinque righe per un totale di 20 lettere. Nessuno riesce ad identificare il testo dal quale proviene la scritta fino al 1972, quando il papirologo spagnolo José O’ Callaghan pubblica i risultati di un suo studio ${ }^{94}$ che dimostra come, utilizzando la tecnica della sticometria95, si possano collocare le quattro lettere "nnes" dell'ultima riga all'interno di un brano evangelico. Più precisamente le lettere riguardano il termine "Gennesaret", nome aramaico del lago di Tiberiade, ed appartengono ai versetti 6, 52-54 di Marco delle edizioni greche. ${ }^{96}$ Una controprova effettuata con lo stesso metodo su un altro frammento della grotta, mostra chiaramente un brano della prima lettera di Paolo a Timoteo. Si aggiunga che tutti i papirologi, pur senza conoscere il significato ma in base al tipo di scrittura ed altre particolarità grafiche, avevano già datato il frammento intorno al 50 d.C.

L'effetto tra gli studiosi è dirompente, perché viene anticipato di almeno due decenni il vangelo di Marco, ed ancor più se si tiene conto che l'originale in aramaico non poteva che precedere il testo

94 J. O’Callaghan, Papiros neotestamentarios en la cueva 7 de Qumran? In Biblica 53, 1972, pp. 91-110.

95 Sticometria, procedimento con cui si calcolava la lunghezza delle opere antiche in base ad un numero prefissato di sillabe o lettere per riga, utilizzato come unità di misura necessaria a fissare la remunerazione del copista.

96 Marco, 6, 52-54 "[...] Compiuta la traversata approdarono a Gennesaret [...]". 
tradotto in greco: si tratta del più antico manoscritto conosciuto del Nuovo Testamento.

Ma la grotta Q7 riserva un'altra sorpresa: viene rinvenuta anche un'anfora in frantumi che conteneva documenti, con impressa due volte sul collo l'iscrizione in ebraico $R W M$ (che si legge Ruma o Roma), ed è appurato che il nome indichi la provenienza. Ciò sta a significare che Marco ha scritto il suo vangelo a Roma, al seguito di Pietro come la tradizione ha sempre indicato. Significa anche che tra gli esseni ritiratisi nel deserto esisteva una comunità cristiana, che aveva ricevuto da quella romana una copia di quello che si considera il primo vangelo composto.

Il papiro Magdalen deriva il suo nome dal Magdalen College di Oxford, che lo conserva. Tre frammenti del papiro sono stati donati al Magdalen dal rev. Charles Huleatt, in gioventù allievo del college, che li aveva acquistati in Egitto. Huleatt li aveva correttamente identificati come brani del vangelo di Matteo e datati all'inizio del IV secolo. Dopo decenni di oblio, nel 1953 Colin Roberts ne anticipa la datazione al II secolo, cosa che viene accettata senza contestazioni. Nel 1994 lo studioso e papirologo Karsten Thiede dimostra però, dopo approfondite analisi effettuate con l'ausilio di avanzate tecniche scientifiche, che la datazione deve essere anticipata al 65-66 d.C. ${ }^{97}$ E' una datazione che verrà confermata dal ritrovamento di un altro papiro, scritto con lo stesso stile e grafia e con data certa, che contiene la seguente data «il dodicesimo anno dell'imperatore Nerone Claudio Cesare Germanico»,98 cioè il 66 d.C. Accettare che, oltre Marco, anche Matteo abbia scritto prima della distruzione di Gerusalemme anticipa la stesura anche degli altri due vangeli, mette in discussione posizioni consolidate, e provoca anche in questo caso la dura ma non documentata reazione degli "addetti ai lavori".

97 K. Thiede, M. D’Ancona, Testimone oculare..., op. cit., p. 78 sgg.

98 M.Astrua, La storicità del vangeli, una guerra vinta, Pessano con Bornago, 2009, p. 28-30. 


\section{LA SINDONE E LA SUA STORIA}

Si tratta dell'oggetto archeologico più studiato al mondo, sul quale sono stati scritti centinaia di libri e notizie quasi quotidiane sui media di tutto il mondo. Basti dire che il primo libro sulla Sindone è stato scritto nel 1598, pochi anni dopo il suo trasporto a Torino. La particolarità che lo rende unico, oggetto di venerazione ma anche di aspre contese storico-scientifiche, è quella di essere ritenuto il sudario che ha avvolto e custodito il corpo di Gesù dopo la deposizione dalla croce. Si tratta di un tessuto di lino con trama a spina di pesce, all'epoca di particolare pregio, della misura di circa 4,40x1,10 mt. (originariamente 8x2 cubiti siriani). Quanto da secoli attira l'attenzione e suscita pietà, interesse o diffidenza, è ciò che la Sindone mostra impresso: la sagoma di un uomo disteso, con evidenti tracce di morte violenta.

Sul telo si notano diverse impronte, non facilmente comprensibili al primo sguardo. ${ }^{99} \mathrm{Si}$ possono osservare:

- l'immagine frontale e dorsale di una sagoma umana, composta in atteggiamento di grande serenità;

- macchie di sangue in corrispondenza delle ferite e lesioni a vario titolo riportate dall'uomo avvolto, ed un ampio alone di sangue e siero in corrispondenza della ferita al costato, che prosegue sul dorso;

- fori, aloni e macchie, causati sia dall'incendio subito a Chambery nel 1532, sia da altri incendi od incidenti.

Le prime notizie documentate che possediamo della Sindone risalgono al 1205 e si ricavano da una lettera di supplica al papa

99 Per comodità descrittiva si farà riferimento, salvo specifica menzione, alle dettagliate fotografie sindoniche. La straordinaria ed inspiegata particolarità del telo è quella di essere un vero negativo fotografico, con rappresentazione speculare e invertita del corpo. Il telo però non è di facile diretta lettura, a causa dello scarso contrasto dell'immagine. 
Innocenzo II inviata dal nipote dell'imperatore Isacco II Comneno, in cui si esprime lo sdegno per il saccheggio di reliquie, prima fra tutte la Sindone, effettuato da crociati e veneziani a Costantinopoli l'anno precedente. Secondo Comneno, la Sindone è stata portata ad Atene. Si ritiene che sia stata trafugata da Ottone de la Roche che, dopo la fallita crociata ed il sacco di Costantinopoli, viene nominato duca di Atene ed ivi risiede, prima di tornare in Francia e morire nel 1224. Non si hanno informazioni certe fino al secolo successivo; molti ritengono che sia custodita dai Templari, segretamente ad evitare sanzioni pontificie per un delitto punibile con la scomunica. L'ordine dei templari viene comunque soppresso nel 1312 e, tra le figure di spicco messe al rogo, c'è un Geoffroy de Charnay.

La Sindone riappare a Lirey nel 1324 nelle mani del suo feudatario, omonimo nipote del templare Geoffroy de Charnay finito al rogo; ciò può spiegare perché riappaia dopo un secolo in un piccolo paese francese. Gli eredi di Geoffroy conservano la Sindone per un secolo, fra litigi con la collegiata di Lirey che ne rivendica la proprietà e col vescovo di Troyes, con accuse di falso, richieste di scomunica, bolle e contro bolle papali, finché l'ultima erede, Margherita, la cede segretamente a Ludovico di Savoia in cambio del castello e della città di Flumet. Segue un periodo di squallidi mercanteggiamenti fra i canonici di Lirey, Margherita ed i Savoia, fino alla vendita definitiva della Sindone a Ludovico di Savoia da parte dei canonici di Lirey nel 1464. La Sindone resta da allora proprietà di Casa Savoia, che la trasferirà il secolo seguente a Torino, ove resterà, salvo brevi occultamenti per sottrarla a rischi di guerre. Nel 1983 verrà destinata, per legato testamentario dell'ultimo re d'Italia in esilio Umberto II, a papa Giovanni Paolo II e quindi alla Santa Sede, ma lasciata in custodia all'arcivescovo di Torino.

Si può dunque ritenere certa l'esistenza della Sindone nel 1205, ed il suo peregrinare da Costantinopoli ad Atene, in Francia e, successivamente, in Italia. Che si tratti dello stesso telo è confermato anche dalle tracce dei danni subiti nell'incendio di Chambery nella 
cappella che la custodiva: si tratta di una serie di fori provocati da gocce d'argento fuso della teca che la conteneva, e degli aloni dell'acqua utilizzata per spegnere il fuoco.

Abbiamo parlato del cammino della Sindone in Europa, a seguito del sacco di Costantinopoli. Ci si pone però, giustamente, la domanda di quali siano state le vicissitudini di questo telo nei secoli intercorsi tra il 30 d.C. ed il suo apparire nel 944 a Costantinopoli, dove verrà custodita fino al 1204, quando la Sindone viene trafugata.

E' arduo stabilire chi abbia raccolto e conservato la Sindone nei primi decenni seguenti la morte di Gesù, ed i racconti e le più varie credenze si intrecciano. Occorre partire da alcuni punti fermi. Il sudario di Gesù è qualcosa di estremamente imbarazzante e pericoloso: per gli ebrei è un oggetto impuro che ha avvolto il cadavere di un giustiziato, e non è pensabile che siano i Sacerdoti a conservarlo; per i romani è qualcosa di disonorevole perché testimonia una crocifissione, e ben poco interessa loro un telo sporco e macchiato di sangue; scartata anche l'improbabile consegna alla moglie di Pilato, restano i discepoli. E' vero che questi sono ebrei, vincolati ai precetti che riguardano il trattamento dei cadaveri e da quanto da essi contaminato, ma è altrettanto è più vero che si tratta di tutto ciò che di tangibile hanno di Gesù, il loro Rabbi. Inoltre, se è vero che Gesù è apparso loro in carne ed ossa più volte, come non pensare che le remore ed i precetti ebraici si affievoliscano? Non è allora irragionevole credere che siano loro a conservare gelosamente ed in segreto il lenzuolo funerario. Se infine aggiungiamo il destino di Gerusalemme, la sua duplice spietata distruzione ad opera dei romani e la conseguente diaspora del popolo ebraico, si comprende perché chi detiene quello che considera un tesoro inestimabile si muova con circospezione. E' allora ingenuo o poco sensato accampare la mancanza di attestazioni certe in un periodo che ha vissuto la fine del mondo ebraico, le persecuzioni ai cristiani, le invasioni barbariche, la caduta dell'impero romano d'occidente, la nascita 
e l'espandersi dell'Islam. Un mondo, cioè, che ha visto affievolire e poi dissolversi la pax romana e le sue regole, dure ma portatrici di equilibrio e pace sociale. Malgrado tutto ciò la Sindone ha lasciato tracce ed indizi nel percorso che l'ha portata al suo approdo a Costantinopoli nel X secolo.

Fuggendo da Gerusalemme, oggetto della distruzione romana del 70 d.C., i giudeo-cristiani prendono dapprima la via di Pella, nella Decapoli, dalla quale poi, dopo la distruzione della città nel 131 d.C. operata dai romani a seguito della rivolta capeggiata da Bar Kocheba, raggiungono Edessa. La città è la capitale del regno indipendente di Oshroène, situato tra impero romano e Persia, ove la lingua parlata è lo stesso aramaico di Gerusalemme. Un'icona del $\mathrm{V}$ secolo mostra la presentazione al re Agbar del telo sindonico da parte dei profughi al loro ingresso in Edessa: il re dispiega sulle ginocchia un lungo lino che mostra l'impronta di un viso simile a quello della Sindone. Va ricordato che a Edessa viene edificata quella che è ritenuta la prima chiesa cristiana, e che nel 170 il regno di Oshroène si dichiara ufficialmente cristiano.

Nel 212 Caracalla occupa Edessa e rovescia la dinastia, creando la Colonia Edessenorum. ${ }^{100}$ Intanto il cristianesimo è divenuto nemico politico dell'impero e viene perseguito, con la comunità cristiana che sprofonda nella clandestinità. Secondo la tradizione il telo "Acheiropoietos" (non dipinto da mano d'uomo) viene nascosto in un" alta nicchia delle mura, dove resterà, fino a perderne le tracce, per oltre tre secoli.

Edessa è sulla strada di tutte le battaglie tra i romani d'occidente e poi d'oriente ed i persiani, che si alternano nel controllo della città. Nel 544 i persiani, con Cosroe I, assediano la città ed iniziano la demolizione delle mura, quando accade l'imprevisto: viene rinvenuta una nicchia murata che racchiude il telo. Gioia ed emozione esplodono tra gli assediati, che percorrono osannanti le

100 M.G.Siliato, Sindone, op.cit., p.164. 
mura dispiegandolo. I persiani osservano con superstizioso timore, che si trasforma in terrore quando il forte vento alimenta un incendio che distrugge accampamento e macchine da guerra. Cosroe è costretto a togliere l'assedio e l'anno seguente firma la tregua con Giustiniano. Conosciamo questi avvenimenti perché vengono descritti con precisione da Evagrio Scolastico, prefetto imperiale di Antiochia e storico di valore.

La ritrovata conoscenza del viso modificherà i canoni artistici fino ad allora seguiti, che mostravano Gesù con capelli corti e lineamenti giovanili, adeguando rapidamente ritratti ed incisioni alla figura impressa. Questa è inequivocabilmente rappresentata nelle monete bizantine coniate dal VI secolo in poi. Giustiniano fa edificare una chiesa sontuosa, che chiama Haghia Sophia (come quella di Costantinopoli), nella quale viene conservato il telo ripiegato in modo da mostrare il solo viso, celando il corpo nudo e martoriato inaccettabile per gli antichi cristiani. ${ }^{101} \mathrm{Ma}$ Edessa non ha pace, e viene conquistata dagli arabi nel 638. Un patto di vassallaggio la risparmia dal saccheggio; il culto viene concesso con forti limitazioni, soggetto all'arbitrio di sovrani o governatori fanatici. L'esposizione ed il culto del Telo, pur rispettato, diverranno sempre più prudenti e difficili nei tre secoli che seguiranno.

Nel 944 l'imperatore bizantino Romano Lecapeno decide che il Telo venga tolto al controllo degli arabi, e conservato a Costantinopoli. Dietro minaccia di assedio, tratta con il califfo $\mathrm{Al}$ Muttaqi la consegna del telo, convincendolo con una generosa offerta. Malgrado l'opposizione della comunità cristiana edessena, la reliquia giunge a Costantinopoli il 15 agosto nel tripudio generale, con la famiglia imperiale ad attenderla: una miniatura

101 La piegatura verrà denominata "Tetradiplon" (quattro volte doppio) e fa sì che emerga il solo volto. Recenti foto della Sindone fatte a luce radente hanno evidenziato tracce di piegatura che si accordano con le descrizioni della tradizione (J. Wilson, British Society for Turin Shroud "Icone ispirate alla Sindone, testimonianze del VI secolo"). 
del Codice Skylitzes, della Biblioteca Nazionale di Madrid, mostra il Telo dispiegato tra le braccia dell'imperatore, che lo bacia devotamente. In un' omelia dell'anno seguente, il dignitario incaricato di tenere i rapporti tra l'imperatore ed il patriarcato, Gregorio il Referendario, parla dell'impronta portata da Edessa «impressa delle sole gocce dell'agonia[...]ulteriormente abbellita dal sangue sgorgato dal suo stesso fianco».

C'è infine un altro importante documento del XII secolo, il " $\mathrm{Co}_{0}$ dice Pray" della Biblioteca Nazionale Széchényi di Budapest, che mostra due immagini inequivocabili: la prima mostra l'unzione del corpo di Gesù, che presenta caratteristiche simili a quelle della Sindone; la seconda mostra la scoperta nella tomba del lenzuolo funebre, con la stessa inconsueta tessitura a spina di pesce e quattro fori disposti a L, che anche la Sindone presenta, forse tracce di un'antica bruciatura da cera fusa.

E' quindi un percorso tormentato quello della Sindone nei primi secoli, almeno fino al suo arrivo a Costantinopoli, fatto di apparizioni ed occultamenti. Certo mancano le incontestabili prove che possediamo da Costantinopoli in poi, ma è innegabile la presenza di indizi, testimonianze, notizie e raffigurazioni che giuridicamente si definiscono seri, ripetuti, precisi e concordanti, e che ben si saldano al periodo francese ed italiano, assicurando, al di là di ogni ragionevole dubbio, continuità e piena attendibilità storica.

\section{ix. Raffronti tra Sindone e narRato eVANGelico}

Quanto verrà esposto non parte dai singoli episodi evangelici allo scopo di cercare conferme nel telo sindonico. Il viaggio è inverso: partire da quanto appare e procedere a ritroso, verificando se esiste compatibilità tra quanto si può rilevare sulla Sindone e quanto i vangeli dicono, oppure se la loro spesso scarna descrizione può essere ragionevolmente integrata da quanto la Sindone mostra. Parlando in termini giuridici è dimostrare se tale reperto, ritrovato dopo 
secoli di occultamenti e traversie, sia appartenuto all'uomo Gesù, crocifisso venti secoli fa a Gerusalemme secondo modalità proprie dell'occupante romano, come dai vangeli narrato.

Occorre ricordare che la Sindone è di non facile interpretazione alla diretta osservazione, a causa dello scarso contrasto dell'immagine col tessuto che va ingiallendo col tempo. E' stato solo a partire dalla prima fotografia, scattata nel 1898 dall'avvocato e fotografo dilettante Secondo Pia, che si sono evidenziati, appunto con precisione fotografica, particolari che ictu oculi sono di difficile percezione. E qui parliamo subito di una particolarità straordinaria della Sindone, che sino ad oggi nessuno ha saputo compiutamente dimostrare o ripetere: il lenzuolo è un vero e proprio negativo fotografico. E' come se l'immagine che vediamo all'osservazione diretta fosse quella riflessa in uno specchio. Per questo tutti i dati di seguito esposti si riferiscono al corpo dell'uomo, e non a quello che la Sindone mostra a rovescio. Per esemplificare, se parliamo del braccio sinistro si tratta del braccio dell'uomo, che sulla Sindone appare come destro. Che non si sia trattato di errori di un fotografo dilettante, alle prese con una tecnica in via di sviluppo, verrà confermato dalle fotografie ufficiali del 1931 e 1969 (per la prima volta a colori), oltre alle migliaia scattate con attrezzature d'avanguardia da studiosi e scienziati di ogni branca.

Per facilità di lettura, la terminologia tecnica e le spiegazioni mediche e scientifiche saranno ridotte al necessario, rispettandone però significati e conclusioni. Ogni più ampio approfondimento è comunque ricavabile dalle opere a nota citate.

Giò che il lenzuolo sindonico mostra all'osservatore, seppure con i limiti sopra descritti, sono le impronte anteriore e posteriore, contrapposte per il capo, di un uomo disteso in posizione supina, con le mani incrociate sul pube, i piedi sovrapposti e la testa reclinata in avanti. Viene calcolata una statura intorno ai $180 \mathrm{~cm}$., un peso 
stimato di $75 \mathrm{Kg}$; l'età che si deduce dall'impronta del volto e del corpo è quella di un uomo di circa 40 anni, di vigorosa struttura. ${ }^{102}$

La lastra sepolcrale sulla quale è stato disteso il corpo avvolto nel telo è stata probabilmente cosparsa da un consistente strato di aromi, che ha creato una sorta di morbido cuscinetto. Lo si deduce dal fatto che l'impronta dorsale non mostra appiattimenti delle parti prominenti (glutei, polpacci, spalle). E' agevole il riscontro col Vangelo di Giovanni, in cui appare Nicodemo al momento della deposizione e sepoltura, che porta 100 libbre di aloe e mirra, con evidente funzione di contrastare la decomposizione del cadavere ed il conseguente cattivo odore che avrebbe emanato. Va ricordato che il corpo non è stato lavato od unto, cosa non prevista dal rituale ebraico per questo tipo di morte. Se lo si fosse fatto si sarebbero perse tutte le tracce ematiche che sono invece presenti sul telo.

Sulla parte destra del torace è presente un'ampia macchia di sangue, di circa $6 \times 15 \mathrm{~cm}$, che si estende alla parte posteriore, all'altezza dei lombi. E' procurata da una ferita al costato, con caratteristiche di un colpo di lancia inferto dal basso verso l'alto. Si tratta di sangue e liquido acquoso, ben distinti nelle macchie impresse sul tessuto. ${ }^{103}$ E' conferma che l'uomo della Sindone è stato colpito a morte avvenuta, come confermato alla lettera da Giovanni. ${ }^{104}$ In corrispondenza della fronte, del cuoio capelluto e della nuca si rilevano numerosi segni puntiformi, dai quali scendono rivoletti di sangue. Ai lati della fronte si osservano due colature con diversa inclinazione, corrispondenti alle diverse inclinazioni del capo durante l'agonia. Al centro

102 P. L .Baima Bollone, Sindone, storia e scienza, Ivrea, 2010, pp. 185-190.

103 Idem, Sindone...op. cit., p. 193. "Al momento del ferimento è uscita la parte corpuscolata rossa, e successivamente quella sierosa-acquosa, fenomeno definito dissierazione. E' estremamente significativo, perché ciò si realizza solo dopo la morte".

104 Giovanni, 19, 33-34, “[...] quando (i soldati) videro che era già morto non gli ruppero le gambe, ma uno dei soldati con la lancia gli aprì il costato; e subito ne uscì sangue ed acqua". 
spicca un rivolo, che ha assunto la forma di un 3 per il corrugamento della fronte causato dall'intenso dolore. La distribuzione delle lesioni sul capo porta a considerare che si sia trattato di una sorta di corona calzata a forza in testa. Pure qui abbiamo conferma nei vangeli, che parlano di una corona di spine. ${ }^{105} \mathrm{Il}$ volto mostra segni di numerose lesioni traumatiche: tra le arcate sopraccigliari si notano ferite; il naso è ferito, tumefatto e presenta una deviazione causata da trauma; la parte destra del viso è pure tumefatta ed è solcata da due rivoli di sangue usciti dalla ferita nasale. Anche le ginocchia appaiono escoriate e contuse. ${ }^{106}$ Verificando $i$ vangeli è agevole il riscontro con la descrizione degli schiaffi, delle percosse e delle bastonate sulla testa ricevute dai soldati. E' verosimile, anche se i vangeli non ne parlano, il ripetersi di percosse durante la via crucis, e l'effetto di cadute in avanti sotto il peso del patibulum. Proprio al patibulum si legano due macchie quadrangolari nella parte dorsale, prodotte dall'oscillazione della trave trasportata che provoca escoriazioni sulle zone scapolari. ${ }^{107}$ Non si tratta però di lesioni dirette, ma presuppongono la presenza di qualcosa tra il corpo ed il legno: tutto combacia con quanto descrivono gli evangelisti, di Gesù che viene rivestito dai soldati e caricato del patibulum.

Sulla parte frontale dell'immagine, ma soprattutto sul dorso, appaiono decine di piccoli segni tondeggianti simili ad un'eruzione cutanea. I vari medici legali che analizzavano le foto avevano ipotizzato trattarsi di qualcosa mai vista su un corpo vivente o su raffigurazioni: gli effetti di una flagellazione. Nel 1976 le analisi effettuate a Pasadena con strumentazione avanzata daranno conferma inequivocabile: i segni sono piccole ferite tondeggianti, riunite a due a due. Si tratta di lacerazioni quasi invisibili ad occhio nudo, ma sufficienti a stillare sul tessuto una piccola quantità di sangue e

\footnotetext{
105 Matteo, 27, 29; Marco, 15, 17; Giovanni, 19, 2; 19, 5.

106 P. L. Baima Bollone, Sindone..., op. cit., pp.195-196.

107 P. Barbet, La Crocifissione e la Sindone, New York, 1995.
} 
siero. E' l'effetto di una flagellazione effettuata col flagrum taxillatum in uso alla truppe romane, impugnato da due esecutori. Si contano circa 120 colpi di sferza, ma il conteggio sale considerando che le parti laterali del corpo non appaiono sul telo. Anche in questo caso ciò che la Sindone evidenzia trova precisa conferma nei vangeli, che parlano esplicitamente della flagellazione subita da Gesù. ${ }^{108}$

Le rappresentazioni pittoriche hanno quasi sempre mostrato Gesù crocifisso con chiodi nei palmi, ed i piedi sovrapposti trafitti da un solo chiodo. La Sindone mostra senza equivoci i piedi trafitti nei calcagni, con ampie colature di sangue sul dorso e sulla pianta di ambo i piedi. La maggioranza degli studiosi propende per un unico chiodo, e solo pochi parlano di due chiodi. Una conferma del chiodo unico e del crurifragium è giunta da una tomba scoperta nel 1968 nei pressi di Gerusalemme, contenente l'urna datata intorno al 70 d.C. di un uomo, Jehohanan Ben Hgqwl, sottoposto ad evidente crocifissione $^{109}$. Nel calcagno destro era rimasto infisso il chiodo che aveva trapassato entrambi i talloni, con la punta ritorta e frammenti di legno d'ulivo. In più tibia e perone mostravano i segni di una violenta frattura: il crurifragium.

La grande sorpresa della Sindone è stata mostrare i segni dei chiodi infissi nei polsi, pratica confermata dai resti del crocifisso del 70 d.C., il cui arto superiore mostrava le abrasioni ossee provocate da un chiodo infisso tra ulna e radio ${ }^{110}$, e lunghe colature di sangue che dagli avambracci risalgono verso i gomiti, cosa dovuta alla posizione del crocifisso, con le braccia tese verso l'alto per il peso del corpo.

Il primo a dare spiegazione dei chiodi nei polsi e non nei palmi è l'anatomista Pierre Barbet, che dimostra con esperimenti su cadaveri come i palmi non reggano il peso di un corpo, ed individua

108 Mattteo, 27, 26; Marco, 15, 15; Giovanni, 19, 1.

109 B. Barberis, M.Boccaletti, Il caso Sindone non è chiuso, Cinisello Balsamo, 2010, pp. 120-123.

110 M. G. Siliato, La Sindone, op.cit., pp. 292-293. 
il punto nel cosiddetto "spazio di Destot", nel polso."1' In quello spazio il chiodo si inserisce senza causare fratture, ed i polsi reggono un corpo sospeso. Simili esperimenti verranno ripetuti da altri anatomisti, tra i quali Judica Cordiglia, con identici risultati. Evidentemente ai romani questo era noto per esperienza e pratica ripetuta..

Quanto alla stranezza che sulla Sindone non appaiano i pollici, ciò si spiega con la lesione del nervo mediano, causata dall'ingresso del chiodo, che provoca la ritrazione del pollice.

I vangeli non parlano di palmi o polsi, ma genericamente di mani e chiodi. ${ }^{112}$ La crocifissione con l'uso di chiodi, che la Sindone mostra, trova quindi piena conferma nei vangeli; in più fornisce un'informazione che, pur da essi non riportata, non li contrasta, anzi li arricchisce di nuovi particolari: l'inchiodatura nei polsi.

\section{LA scienza dove L'occhio NON PUò VEDERE}

Nel paragrafo precedente si è esaminato ciò che la Sindone mostra, direttamente od attraverso le più nitide fotografie. Ma le sue sorprese non sono terminate: cinquant'anni di ricerca scientifica hanno portato novità, spesso sconvolgenti ed invisibili alla diretta osservazione, che hanno fatto conoscere caratteristiche impensate del telo e dell'uomo avvolto, aggiungendo loro ulteriore veridicità. Il raffronto coi vangeli diviene ovviamente impossibile, ma quello che la scienza ha scoperto nulla toglie alla loro narrazione, anzi, li rafforza nell' autenticità e credibilità.

Uno dei problemi che ci si è posti è stato quello di verificare se quelle che sul telo appaiono come macchie di sangue in realtà lo siano, e riuscire a determinarne le caratteristiche. Utilizzando fili macchiati del telo e minuscole croste da polveri aspirate, Baima Bollone

111 P. Barbet, La Passion..., op. cit.

112 Giovanni, 20, 24-29 "[...] ma egli (Tommaso) oppose loro: se non vedo nelle sue mani il segno dei chiodi e non metto il mio dito al posto dei chiodi, non crederò". 
è riuscito con l'ausilio delle più avanzate apparecchiature prima a chiarire che il sangue che macchia il telo è di natura umana, in seguito a determinarne il gruppo sanguigno in $\mathrm{AB}$. Questo è poco frequente in Europa (circa il 5\% della popolazione), mentre tra gli ebrei è assai più diffuso, e raggiunge il $20 \%$. Dagli esami appaiono significativamente anche tracce di aloe e mirra. ${ }^{113}$ Questi esami sono stati ripetuti e confermati da altri ricercatori indipendenti ${ }^{114}$.

Quanto alla ricerca del DNA gli studi sono in corso, complicati dalla scarsità del materiale da analizzare e dalle innumerevoli tracce lasciate da chi per secoli ha maneggiato la Sindone. Gli studi più accreditati sono ancora di Baima Bollone in collaborazione con un'équipe scientifica dell'Università di Genova, e quelli dell' Università di San Antonio nel Texas, ${ }^{115}$ che confermano trattarsi di sangue umano molto antico. Un'altra ricerca, effettuata dal team del prof. Gianni Barcaccia presso il laboratorio di genetica dell'Università di Padova, ha identificato nelle polveri sindoniche numerose particelle di origine umana, «appartenute a più individui geneticamente diversi, tutti riconducibili a gruppi europei, nordafricani e mediorientali, soprattutto a popolazioni di paesi che si affacciano sul Mediterraneo». ${ }^{16}$ E' un dato che pone una difficoltà in più a chi contesta la provenienza mediorientale della Sindone, attribuendole un'origine centroeuropea.

Tra le diverse analisi effettuate sulle polveri sindoniche, c'è stata la ricerca di pollini della flora presente lungo il cammino della Sindone. I granuli di polline hanno la caratteristica di resistere ad acidi ed alcali, al calore ed al gelo, sì che è possibile identificarli

113 P. Baima Bollone, Sindone..., op. cit., pp. 230, 233-234.

114 Fra i tanti, B.Barberis, G.M.Zaccone, La Sindone, cento anni di ricerca, Roma, 1998, pp. 165-184; L.Garza Valdes, The DNA of God, New York, 1991, p. 19.

115 O.Petrosillo, E. Marinelli, La Sindone, storia di un enigma, Milano, 1998, pp. 85-86.

116 G. Fanti, S.Gaeta, Il mistero della Sindone, Milano, 2013, pp. 186-187, che citano la ricerca del prof. Barcaccia. 
anche a distanza di migliaia di anni. La scienza che se ne occupa è la palinologia.

I più importanti studi sono stati effettuati dal ricercatore Max Frei, svizzero calvinista, affermato palinologo e consulente di polizie e tribunali. Nel 1973 e nel 1978 viene incaricato di effettuare prelievi sul telo, che realizza grazie a speciali nastri adesivi che inglobano le tracce presenti. All'epoca delle fioriture Frei raccoglierà campioni a Gerusalemme e sui monti della Giudea, ad Urfa (l'antica Edessa), a Cipro, Costantinopoli, nel nord est della Francia ed in Italia settentrionale. Il materiale raccolto gli consentirà di stilare una mappa dei luoghi e delle piante che riguarda 58 pollini raccolti. Secondo Frei esiste una sola zona in cui crescono 45 di queste piante: è l'area circostante Gerusalemme. Delle altre piante, due crescono esclusivamente ad Urfa/Edessa; una nella zona di Costantinopoli; le altre crescono tuttora in Europa (nel 1560 la Sindone è esposta a Vercelli, zona di risaie: tra i pollini europei identificati figura quello del riso). Frei muore improvvisamente nel 1983, lasciando incomplete le sue ricerche. I lavori sino ad allora effettuati sono stati oggetto di studi e verifiche specialistiche e finora nessuno è riuscito a scalfire le sue valutazioni che, anzi, hanno ottenuto conferme e rivalutazioni.

Ma altri particolari e sorprese ha riservato la ricerca scientifica: in un congresso di studiosi tenutosi ad Albuquerque nel 1978 viene annunciato che sul volto della Sindone si osservano «forme simili a bottoni appoggiati sulle palpebre, forse qualche tipo di moneta». Un'identificazione viene proposta da Francis Filas dell'Università di Chicago, che riferisce di aver rilevato nell'orbita destra la scritta $U C A I$ e l'immagine di un bastone ricurvo, il lituus degli àuguri pagani. ${ }^{17}$ La scritta, identificata in TIBERIOU CAISAROS, è riportata su un dilepton lituus circolante tra il 29 ed il 32 d.C. E' un'interpretazione contestata perché avrebbe dovuto essere KAISAROS, finché non viene ritrovata presso un numismatico di Chicago, in

117 P. Baima Bollone, Sindone..., op.cit., pp. 246-247, 261-266. 
un gruppo di quattordici dilepton lituus, una moneta con la scritta CAISAROS. Si tratta dunque di un errore di conio (queste monete venivano battute anche nelle provincie, con frequenti piccoli errori). Nel 1996 Baima Bollone ed i suoi collaboratori identificano, grazie all'elaborazione tridimensionale del volto, un'altra moneta nell'orbita sinistra con la corretta scritta KAISAROS, ma con al centro la figura di un simplum, tazza sacrificale romana. Questa seconda moneta consente un'inoppugnabile datazione, perché contiene anche la scritta $L I S$, cioè il $16^{\circ}$ anno di Tiberio ( $\mathrm{L}=$ anno; $\mathrm{I}=$ dieci; $\mathrm{S}=$ sei), il 29-30 d.C. ${ }^{18}$ Anche l'obiezione che non era usanza degli ebrei porre monete sugli occhi del defunto verrà confutata dal ritrovamento a En Boqeq, Gerico e Gerusalemme, di scheletri del I sec. d.C. con monete all'interno del teschio. ${ }^{19}$

Un' altra delle tracce disseminate nella Sindone riguarda l'esistenza di possibili scritte intorno al volto. I due fisici nucleari francesi A. Marion ed A.L.Courage annunciano di aver individuato alcune scritte grazie alla tecnica microdensitometrica. Si tratta dei termini INNECE (contrazione di in necem = a morte), NNAZAPENUS (nazareno), JESHUA (Gesù, in ebraico), IC (Iesus Christus) e PEZW (attesto, in greco). ${ }^{120}$

E' pensabile sia opera di un exactor mortis, incaricato di verificare la morte del condannato e di identificarlo. Se le verifiche confermeranno le letture della Frale e di Luraschi avremmo, insieme al LIS che si legge sull'occhio sinistro, l'indiscutibile conferma della morte e sepoltura dell'uomo della Sindone, che le scritte indicano in Gesù Nazareno.

Ma quella che si può forse ritenere la più straordinaria, inaspettata scoperta, arriva dalla curiosità e dall'intuizione di uno scien-

118 Idem, pp. 248-250.

119 M. Moroni, F. Barbesino, Apologia di un falsario, Milano, 1997.

120 J. Léveque, R.Pugeaut, Le Saint Suaire, Paris, 2003, pp. 187-189; B. Frale, La Sindone di Gesù Nazzareno, Bologna, 2009; G.Luraschi, La Sindone alla luce della ragione, Chiavenna, 2010, p. 34. 
ziato statunitense. Osservando nel 1975 le fotografie della Sindone del 1931 e considerando che l'impronta era più intensa dove, secondo logica, il telo aveva aderito al corpo, oppure spariva dove il telo era distante, a John Jackson, fisico matematico che lavorava ai raggi laser ad Albuquerque, venne in mente di utilizzare per quelle immagini la tecnica applicata alle fotografie satellitari, in grado di trasformare fotografie piatte in immagini in rilievo trasformando le variazioni di intensità in misure di distanza tra $i$ vari punti. Insieme con Don Devan, ebreo ortodosso, diede il via al più stupefacente progetto di lettura computerizzata mai tentato di un oggetto archeologico: a ciascuna delle diverse gradazioni di colore venne assegnato un valore di altezza in base alla distanza calcolata dal microdensitometro di Devan, che portò a 65.536 valori di grigio. Nell'impresa di leggere, decifrare e trasformare i chiaroscuri in immagine tridimensionale vennero coinvolti i maggiori specialisti mondiali di analisi dell'immagine del Sandia Laboratory di Albuquerque, che utilizzarono il segretissimo analizzatore di immagini VP8. Quanto l'apparecchio ebbe finito il suo lavoro, spuntò sullo schermo la sagoma plastica di un corpo umano disteso. ${ }^{121}$ Lo stupore fu indescrivibile, paragonabile a quello di Secondo Pia all'apparire della prima lastra fotografica, e pure in questo caso si pensò a possibili anomalie.

L'esperimento venne ripetuto sulle fotografie di due accurate copie pittoriche della Sindone, ma ne uscirono immagini distorte, al pari di altre pitture analizzate. Un ulteriore esperimento, eseguito su alcune fotografie più recenti della Sindone, produsse invece dei risultati ancora più accurati e ricchi di particolari. A quel punto si unirono al gruppo i tecnici del fet Propulsion Laboratory di Pasadena, dove erano state elaborate le foto di Venere e Mercurio scattate dalla sonda Mariner, con l'ausilio del loro gigantesco IBM 365. Fu un lavoro difficilissimo di ripulitura dei segni, macchie d'acqua,

121 M.G.Siliato, Sindone, op. cit., pp. 55-61. 
rappezzi e, soprattutto, dei segni di ordito e trama della tela che si confondevano con l'impronta. Ne emerse una figura come posata senza peso sulla stoffa. Non si notava alcuna direzionalità, cosa che avrebbe implicato l'esistenza di una pittura. Il viso mostrava i traumi subiti: lo zigomo destro tumefatto e la palpebra gonfia; il naso escoriato e la cartilagine nasale distorta e distaccata dall'osso; la fronte tumefatta sopra il sopracciglio sinistro, così come la guancia e le labbra. ${ }^{122}$

Veniva così a cadere l'ipotesi pittorica della Sindone, che per secoli ha rappresentato la spiegazione principale della formazione dell'immagine sindonica.

Si conoscono almeno una cinquantina di copie pittoriche, alcune di discreta fattura, altre copie dichiarate, messe a contatto con l'originale per farne un'icona a scopo devozionale. Tutte hanno in comune il fatto di essere dipinte, ma ogni dipinto ha la sua direzionalità e coloranti facilmente identificabili: sulla Sindone sono stati effettuati test fisici e chimici di ogni genere, ma non è emersa alcuna traccia di coloranti. Neppure il tentativo di aggredire l'impronta con solventi ha prodotto risultati: il colore della Sindone, in realtà, si può definire un "non colore". Un'altra insormontabile difficoltà è data dalla visibilità dell'impronta, che perde nitidezza più ci si avvicina, fino a confondersi col colore di fondo a meno di due metri di distanza. Come avrebbe potuto un pur geniale falsario medievale dipingere la figura? Inoltre, come avrebbe potuto dipingere l'immagine dorsale di un uomo in posizione supina?

Ma altri problemi avrebbe avuto questo geniale artista medievale: come apporre il sangue di un gruppo sanguigno raro, che restasse inalterato nei secoli; come distinguere il sangue dal siero; come non impregnare di colore le fibre del tessuto. Avrebbe dovuto prevedere centinaia di piccoli segni effetto del flagrum taxillatum e della corona di spine; avere ampie conoscenze numismatiche; es-

122 M. G. Siliato, Sindone, op.cit., pp. 63-64. 
sere esperto di fotografia secoli prima della sua invenzione e quindi dipingere un negativo fotografico; disseminare il telo di pollini ed aromi mediorientali; porre sui talloni e sulle ginocchia residui di terriccio identificato come proveniente dalla zona di Gerusalemme; dipingere una figura tridimensionale; inventare una crocifissione ai polsi ed il trasporto del solo patibulum, andando contro secoli di tradizione pittorica.

Alcuni hanno sostenuto trattarsi di Leonardo, dimenticando però che il genio toscano è vissuto oltre cinque secoli dopo il trasporto della Sindone a Costantinopoli... Resta poi sempre da fare una considerazione, banale ma essenziale: con tutte queste conoscenze ed accortezze perché mai un tale genio avrebbe dovuto usare un telo coevo, facilmente identificabile?

Scartata obtorto collo l'ipotesi pittorica, gli irriducibili negazionisti dell'autenticità si sono cimentati in altre prove. L'antropologo Vittorio Pesce Delfino ha tentato di dimostrare che l'immagine sarebbe stata ottenuto applicando il telo su un bassorilievo riscaldato a $220^{\circ} .{ }^{123}$ A parte i problemi di maneggiare un bassorilievo di centinaia di Kg. surriscaldato, le obiezioni sono le stesse viste per la pittura, con l'aggiunta di due: la prima è che l'impronta sindonica è superficiale ed interessa solo due o tre fibrille, mentre qui trapassa il tessuto e svanisce in pochi mesi; l'altra è come posizionare con esattezza le tracce di sangue prima di porre il telo sul bassorilievo riscaldato, senza che il sangue si alteri al calore: posizionarlo a posteriori non è possibile, perché sotto le croste di sangue della Sindone il telo non è colorato. ${ }^{124}$

Alcuni scienziati hanno fatto ricorso a cadaveri cosparsi con polveri od aromi vari; altri spiegano l'impronta come l'effetto di vapori ammoniacali del sangue e del sudore. Per altri ancora si tratta dell'immagine prodotta dal contatto del corpo cosparso di aromi,

\footnotetext{
123 V. Pesce Delfino, E l'uomo creò la Sindone, Bari, 1982.

124 O. Petrosillo, E.Marinelli, La Sindone..., op.cit., pp. 97-99.
} 
come le impronte di foglie e fiori sulle pagine di erbari. L'ultimo tentativo in ordine di tempo è quello di Luigi Garlaschelli, docente di chimica all'Università di Pavia, effettuato nel 2009. Garlaschelli sostiene di aver realizzato una copia perfetta, su tela invecchiata e surriscaldata. Afferma di aver utilizzato come matrici il corpo di un assistente ed un calco in gesso cosparso di sostanze chimiche, ma anche in questo caso le caratteristiche fisiche e chimiche dell'impronta ottenuta sono ben diverse da quelle dell'immagine sindonica.

$\mathrm{Va}$ riconosciuto che ognuna di queste e di altre ipotesi ha prodotto qualche risultato simile a quelli della Sindone, ma che ciascuna è ben lontana dal soddisfarne tutte le caratteristiche, e questo è ancor più significativo tenuto conto del dispiegamento di studiosi e delle moderne conoscenze ed apparecchiature. Diverse teorie sono risultate carenti di verifiche serie: non basta produrre un'immagine somigliante a quella sindonica, ma occorre sottoporre i risultati ottenuti a tutte le prove che su questa sono state eseguite.

Abbiamo dunque esaminato come non è possibile che si sia formata l'immagine; cerchiamo di capire invece come questo possa essere accaduto.

La Sindone è un tessuto di lino i cui fili hanno uno spessore di circa 0,30 mm. Ogni filo è composto da un fascio di 100-200 fibrille cave del diametro di 0,0015/0,0020 mm; quelle che appaiono colorate sono le prime due o tre fibrille esterne, le altre sono bianche. Altra caratteristica è che il colore scompare quando un filo colorato passa sotto un altro filo.

Le diverse ipotesi si scontrano con una serie di obiezioni che le vanificano: l'intensità del colore; capelli e barba non soffici; mancanza di tridimensionalità; tendenza alla putrefazione; fibre strinate od impregnate da varie sostanze; contorni non ben definiti; incapacità di trattare le tracce ematiche; tendenza dell'immagine a svanire rapidamente; utilizzo di sostanze chimiche varie, quali l'acido solforico (prodotto solo dal XVII secolo). 
Oggi l'ipotesi della radiazione è la più seguita, sebbene da un punto di vista scientifico non sia accettabile che il corpo di un morto produca l'energia necessaria a formare un'immagine sul tessuto. ${ }^{125}$ Sono state avanzate ipotesi diverse di radiazione, che vanno da un lampo ultravioletto, ad una sorgente laser, ad una radiazione neutronica emessa al momento della smaterializzazione del corpo, all' "effetto corona" derivante da un'emissione di radon causata da un terremoto, ecc.

Ciascun meccanismo esaminato può giustificare una o più caratteristiche della Sindone, ma non tutte. Spiegazioni sostenibili da un punto di vista della chimica sono escluse dalla fisica e viceversa. E' possibile che alla formazione dell'immagine abbiano concorso più fenomeni, ma non si sa quali e come. Per gli scienziati più attenti si tratta di un mistero che continua, ed il concetto è stato ben espresso da Jackson illustrando la tridimensionalità dell'immagine: «sulla base dei processi fisico-chimici conosciuti avremmo motivi per dire che l'immagine sindonica non può esistere, ma essa è reale, anche se non riusciamo a spiegarci come si sia formata».

Si è visto come esami, ricerche e studi sulla Sindone siano stati effettuati da studiosi di tutto il mondo e di ogni branca, prevalentemente a titolo individuale od in piccoli gruppi. Un diverso e più organico modo di affrontare la Sindone, le sue caratteristiche ed i suoi "misteri" è stato posto in essere da un gruppo composito di ricercatori, con l'intento di operare congiuntamente ed in modo coordinato: si tratta dello STURP, acronimo che identifica un gruppo internazionale di ricerca, costituito allo scopo di esaminare scientificamente ed organicamente la Sindone. Comprende decine di scienziati di tutto il mondo, di varie religioni, atei, agnostici. Nel 1978 ottiene il permesso di eseguire una serie di esami non distruttivi e di poter prelevare alcuni campioni da sottoporre ad esami di laboratorio. La Sindone viene messa a disposizione degli scienzia-

125 G. Fanti, S. Gaeta, Il mistero...,op. cit., p. 151. 
ti, e di un gruppetto di studiosi italiani con un loro programma di ricerca, per 120 ore consecutive. Le principali conclusioni dello STURP vengono anticipate nel 1981 ed evidenziano:

1. l'assoluta mancanza di pigmenti, pitture, tinture e coloranti sulle fibre del tessuto;

2. che la colorazione dell'immagine è dovuta ad ossidazione e disidratazione delle fibrille del lino;

3. che l'analisi computerizzata dell'immagine evidenzia un'informazione tridimensionale;

4. la mancanza di tracce di unguenti, olii o di qualsiasi secrezione corporea;

5. un contatto tra corpo e telo, con macchie di sangue composte da emoglobina ed albumina;

6. che nessun metodo fisico e chimico conosciuto è in grado di spiegare tutte le caratteristiche dell'immagine;

7. che si può concludere che l'immagine della Sindone è quella di un corpo umano flagellato e crocifisso.

Nel 1985 lo STURP presenterà una serie di proposte volte ad ampliare e perfezionare quanto emerso dal suddetto studio, compresa la datazione col radiocarbonio da effettuare insieme ad un'altra serie di ricerche chimiche e fisiche, ma la richiesta non verrà presa in considerazione. Anzi, purtroppo, lo STURP verrà un po' alla volta emarginato e poi escluso dietro pressione dei sostenitori della datazione col radiocarbonio. ${ }^{126}$

\section{XI.IL FALSO MITO DEL RADIOCARBONIO}

Il carbonio è uno dei più diffusi elementi, presente in tre forme dall'atmosfera all'acqua ed in tutti gli esseri viventi. Quello che ci

126 B. Barberis, M.Boccaletti, Il caso Sindone..., op.cit., pp. 173-177. 
interessa è il $\mathrm{C}^{14}$, che esiste in concentrazione bassissima (si tratta di un atomo ogni mille miliardi degli altri due, per un totale stimato di 10 tonn. su tutto il pianeta). E' radioattivamente instabile ed ha la caratteristica di dimezzarsi ogni 5730 anni. Negli organismi viventi viene continuamente rimpiazzato con l'alimentazione, per cui resta costante; quando l'organismo muore cessano gli scambi con l'esterno, ed il $\mathrm{C}^{14}$ inizia a diminuire secondo le leggi del decadimento radioattivo. Pertanto, misurando la quantità residua si può calcolare il tempo intercorso tra la morte dell'organismo e quello dell' esame. ${ }^{127}$

Semplice? No, perché alcuni fattori rendono difficile o falsano la datazione: questa non è sicura di per sé, ma necessita di una calibrazione dei risultati attraverso verifiche storico-ambientali dell'oggetto da datare; per questo non ha valore di prova assoluta e deve essere affiancata da altri esami. Inoltre i risultati sono notoriamente contrastanti nel caso di periodi brevi ${ }^{128}$ e sono influenzati nel caso di campioni fortemente inquinati dal contatto con l'ambiente nei secoli: i tessuti sono quelli a maggior rischio per l'elevata interazione con l'esterno. La Sindone possiede tutte queste limitazioni; oltre ad essere un tessuto, vari e ripetuti fattori l'hanno fortemente inquinata nel corso dei secoli, falsando i dati della datazione: le traversie del primo millennio; i vari spostamenti; le centinaia di ostensioni che l'hanno esposta a fumo di candele, gocce di cera, baci, strofinio di mani; i funghi e le spore presenti sul tessuto; gli incendi e le secchiate d'acqua per spegnerli; i rammendi delle suore, ecc. Lo stesso Libby, il premio Nobel inventore della radiodatazione, non a caso si era dichiarato contrario a datare la Sindone a causa della sua estrema contaminazione.

127 Idem, Il caso Sindone..., op.cit., pp. 174-177.

128 L. Canfora, Il libro della Frale sull'autenticità della reliquia, La Stampa Torino, 22/7/2009, "Il carbonio 14 per periodi di tempo brevi più che inattendibile è inutile; per oggetti come la Sindone affidarsi a questo esame è ridicolo". 
Ma la chiesa era in quegli anni al centro di attacchi e polemiche, accusata di rifiutare il confronto con la scienza e di evitare la radiodatazione, sostenuta quale unica ed attendibile prova di autenticità. Capifila di questa polemica alcuni laboratori, il direttore del British Museum Michael Tite, il pastore anglicano David Sox , ed Harry Gove, ideatore del particolare metodo di radiodatazione AMS che sarà quello adottato: quindi desiderio di pubblicità e finanziamenti, di fama e gloria.

Le trattative tra il depositario della Sindone card. Ballestrero, che dovrebbe essere assistito dal direttore dell'Accademia Pontificia della Scienze Carlos Chagas (che però escluderà di fatto il cardinale e tutti i suoi collaboratori, gestendo in prima persona ogni rapporto), e le controparti, porteranno alla scelta dei soli tre laboratori che utilizzano il metodo AMS di Gove. Tite ed il British Museum saranno i garanti delle corrette procedure. I test dovranno avvenire alla cieca, ma in realtà i tessuti comparativi forniti da Tite sono totalmente dissimili, per cui il tessuto della Sindone è facilmente riconoscibile. In più, contrariamente agli accordi, viene aggiunto un tessuto risalente con certezza al 1296. Queste dunque le premesse di quella che avrebbe dovuto essere un'operazione scientifica neutrale, di massima trasparenza e garanzia procedurale.

Contrariamente ad ogni logica precauzione il tessuto viene asportato in un solo punto, sul lato inferiore destro dell'immagine frontale, probabilmente il meno indicato: "Il sito prescelto è il peggiore e tra i più esposti a contaminazioni. E' uno degli angoli dai quali la Sindone veniva tenuta durante le ostensioni, a pochi centimetri di distanza da uno dei punti carbonizzati nell'incendio del 1532. Il frammento proviene da un bordo macchiato dall'acqua usata per spegnere l'incendio, dove si sono depositati i prodotti della pirolisi e tutta la sporcizia dei secoli». ${ }^{129}$ Le procedure di taglio sono discutibili: malgrado l'esame debba effettuarsi alla cieca, pur

129 E. Marinelli, Sindone, analisi di un mistero, Milano, 2009, pp. 158-159. 
se cosa già vanificata dai contro campioni che evidenziano quello sindonico, al prelievo partecipano attivamente i responsabili dei tre laboratori incaricati. Prelievo e frazionamento sono effettuati senza guanti né mascherine; le fotografie mostrano il card. Ballestrero coi gomiti appoggiati sulla Sindone che chiacchiera coi vicini, ed una persona con un sigaro in bocca.

Incredibile è il balletto dei pesi e delle misure: il peso del tessuto sindonico era stato calcolato in base al titolo dei fili dal Laboratorio di tecnologia tessile dell'università di Gand e dal National Laboratory di Los Alamos, e determinato tra 20 e 25 mg./cmq., mediamente 23,2 mg. Il pezzo prelevato dalla Sindone, una volta rifilato misura $7 \mathrm{cmq}$, per un peso comunicato di $300 \mathrm{mg}$, cioè $43 \mathrm{mg} / \mathrm{cmq}$ : quasi il doppio! ${ }^{130}$ Ancor più incredibile, Riggi di Numana (che ha effettuato il prelievo), fornirà in una relazione due pesi diversi del frammento prima della rifilatura: 497 e 540 mg. ${ }^{131}$ Peccato che, nel filmato effettuato durante il taglio, il display della bilancia di precisione mostri 478,1 mg.: tre misure diverse per lo stesso frammento!

Le modalità di prelievo sono state talmente disorganizzate, se non peggio, tali da creare comprensibili dubbi sulla validità di quelli che saranno i riscontri di laboratorio.

Gli esami del radiocarbonio $\mathrm{C}^{14}$ nei tessuti vengono effettuati respingendo il controllo o la presenza degli incaricati della Commissione Sindonologica, perché ritenuto atto di sfiducia. Non verranno qui esaminate le procedure da seguire, perché esorbitanti il tema per il loro stretto carattere tecnico. Basti però dire che ancor oggi, a distanza di oltre 25 anni, i laboratori incaricati ed il British $\mathrm{Mu}$ seum non hanno fornito spiegazioni dettagliate, e neppure i criteri, gli standard, le procedure, le verifiche, i verbali. Quel che è emerso

130 M. G. Siliato, Sindone, op.cit., p. 42; O. Petrosillo, E. Marinelli, La Sindone..., op.cit., p. 167.

131 G. Riggi di Numana, Prélèvement sur le Linceul effectué le 21 avril 1988; relazione tenuta al Simposio di Parigi, 7-8 settembre 1989, p. 6. 
non è confortante, e non è garanzia di un lavoro scientificamente e correttamente eseguito: conoscenza preventiva di quel che si doveva esaminare e probabilmente ottenere; contatti ripetuti in itinere tra $\mathrm{i}$ laboratori, con verifica di procedure, di risultati ottenuti e correzione di dati anomali; mancanza di contemporaneità; intromissione costante, anche fisica, di Tite, Sox e di giornalisti a lui collegati; pubblicazione dei dati in una passerella di autocompiacimento e derisione.

La Sindone, con grande pubblicità dell'evento e palese soddisfazione di Tite e dei responsabili di due laboratori, viene datata su un cartello alle loro spalle «1260-1390!».

Tite ne ha ricavato grande pubblicità personale; Sox, con l'aiuto di giornalisti compiacenti, già due giorni dopo l'annuncio ha messo in commercio un velenoso libro in cui annunciava gli esiti degli esami; i laboratori e Gove hanno ottenuto finanziamenti e fondi per la ricerca. Invece la grande maggioranza di scienziati e studiosi, convinta dell'autenticità della Sindone, manifesta sconcerto e sempre maggiori dubbi di fronte ad un risultato inatteso ed incredibile, che cancella anni di studi e ricerche multidisciplinari. Anche alla Santa Sede, ai più alti livelli, c'è incredulità, seppur manifestata nei toni tipici della diplomazia vaticana: con grandi attestazioni di stima e gratitudine il card. Ballestrero viene sostituito per raggiunti limiti di età, e Chagas abbandona l'Accademia Pontificia delle Scienze.

Si poteva pensare che i risultati della radiodatazione avrebbero messo la pietra tombale sopra la Sindone, declassandola a semplice icona. Se questo era l'intento e la convinzione dei "carbonisti", ben presto si sarebbero accorti che avevano ottenuto l'effetto contrario e fornito linfa a nuovi studi e ricerche, che avrebbero portato non solo a disconoscere la validità della radiodatazione effettuata, ma anche a seri e fondati dubbi sull'onestà e correttezza del comportamento tenuto.

Le opposizioni ai risultati sono di vario genere. Molte si appuntano sulle incongruenze di peso tra Sindone e tessuti esaminati, 
insistendo sul fatto che questi provengono dalla parte rovinata dall'incendio, rammendata con la tecnica del rammendo invisibile ma utilizzando fili di altra origine, peso e, naturalmente, del XVI secolo; l'esame sarebbe stato dunque effettuato sui rattoppi e non sul tessuto sindonico. Per altri si tratta dell' insufficiente trattamento di pulizia di un tessuto che ha subito numerose traversie, compreso l'incendio che ha provocato un apporto di carbonio ed il conseguente ringiovanimento. Altri ancora parlano di errori nei calcoli di laboratorio. Alcuni, infine, parlano di giochi di prestigio o, peggio, di frode da parte di Tite e dei responsabili dei laboratori, che avrebbero analizzato il tessuto del 1296.

Molti studiosi e ricercatori hanno utilizzato nuove e più moderne procedure di datazione, utilizzando fili e tessuti residui provenienti dalla rifilatura dei pezzi destinati ai laboratori. I risultati portano a datazioni che mediamente si collocano nel I secolo d.C., con un margine d'oscillazione in più od in meno di 200 anni. ${ }^{132}$

Alla fine, quella che doveva essere la prova regina si sta sempre più mostrando una maldestra e forse fraudolenta operazione, dalla quale hanno iniziato a prendere le distanze alcuni degli autori stessi. Sotto il profilo giuridico e procedurale nessun tribunale degno di questo nome accetterebbe prove e conclusioni simili, frutto di esami di dubbia validità svolti con metodi discutibili e procedure non dimostrate e provate, oggetto di contestazioni precise e documentate da larga parte del mondo scientifico.

\section{xil. Calcolo probabilistico}

Diversi scienziati e matematici si sono cimentati nel calcolare la probabilità che l'impronta della Sindone appartenga ad un uomo crocifisso e che quell'uomo sia Gesù. Bruno Barberis, docente di

132 Per tutti G. Fanti, S. Gaeta, Il mistero..., op.cit., pp. 79-103. 
fisica e matematica all'Università di Torino, ha pure provato, coi risultati che di seguito vengono riassunti. ${ }^{133}$

La sua ipotesi di lavoro consiste nel raffrontare le caratteristiche dell'impronta sindonica col narrato evangelico. Barberis utilizza il calcolo probabilistico, ramo della matematica che assegna un grado di probabilità al verificarsi di un determinato evento, prendendo in considerazione sette significative caratteristiche dell'uomo della Sindone e raffrontandole con quanto di Gesù narrano i vangeli.

1. avvolgimento del cadavere nel lenzuolo: è un fatto raro, perché i crocifissi venivano lasciati in croce e sepolti in fosse comuni: si può pensare che solo 1/100 dei crocifissi abbia avuto regolare sepoltura. Anche Gesù è stato avvolto in un lenzuolo e deposto nella tomba;

2. ferite al capo: sul telo appaiono ferite puntiformi al capo, probabilmente prodotte da aculei; non si ha notizia di simile usanza dei romani o di altri popoli, e prudentemente si può ipotizzare 1/5000. Secondo i vangeli Gesù viene incoronato di spine per dileggio;

3. trasporto del patibulum: l'uomo della Sindone l'ha portato, come si nota dalle escoriazioni sulle spalle: si può assegnare una probabilità di $1 / 2$, perché le crocifissioni erano spesso effettuate su alberi o pali occasionali. Anche Gesù ha portato il suo legno;

4. crocifissione con chiodi: è un trattamento riservato ad esecuzioni che utilizzano patibulum e stipes ed è alternativo all'uso di funi: anche in questo caso si può stimare $1 / 2$ di probabilità. Pure per Gesù vengono utilizzati chiodi;

5. ferita al costato: la Sindone mostra una ferita da lancia al costato, inferta a morte avvenuta; è un fatto poco comune, usandosi di preferenza il crurifragium per accelerare la mor- 
te, e la probabilità è calcolata ad 1/10. I vangeli parlano di Gesù colpito al costato dal soldato, che ne accerta l'avvenuta morte e non lo sottopone a crurifragium;

6. sepoltura frettolosa: il cadavere sindonico è stato avvolto senza lavanda od unzione, cosa che fa ipotizzare un caso particolare; intorno al lenzuolo risultano essere stati posti aloe e mirra. Si tratta di un caso infrequente, cui si può assegnare 1/20 di probabilità. Secondo i vangeli Gesù è stato posto nel sepolcro senza essere lavato ed unto; la mistura portata da Nicodemo aveva quindi solo funzione antiputrefattiva;

7. breve permanenza del cadavere nel lenzuolo: per imprimere il lenzuolo il cadavere deve esserci restato alcune ore, ma non oltre due giorni perché avrebbe avuto inizio la putrefazione. E' cosa già non comune che si deponga il cadavere di un crocifisso nel lenzuolo, ed ancor più è toglierlo prima che inizi la decomposizione: all'evento si può attribuire una prudente probabilità di 1/500. Gesù è stato avvolto nel lenzuolo per circa 40 ore e, nel sepolcro custodito da guardie, si è trovato il solo lenzuolo perché il cadavere non c'era più.

Nel calcolo non viene considerata la flagellazione, perché essendo procedura prevista per il condannato a crocifissione, la probabilità è di $1 / 1$, quindi ininfluente.

La probabilità complessiva che gli eventi descritti si siano verificati nella stessa occasione ed in maniera indipendente tra loro porta al seguente calcolo matematico:

$1 / 100 \times 1 / 5000 \times 1 / 2 \times 1 / 2 \times 1 / 10 \times 1 / 20 \times 1 / 500=1 / 200.000 .000 .000$

cioè su duecento miliardi di crocifissi uno solo poteva possedere tutte le caratteristiche comuni all'uomo sindonico ed al Gesù dei vangeli. Altri studiosi sono giunti a cifre ancor più elevate, calco- 
lando maggiori o differenti caratteristiche comuni tra l'uomo della Sindone e Gesù delle narrazioni evangeliche.

Si può quindi concludere che la probabilità che l'uomo della Sindone sia Gesù di Nazareth è altissima. ${ }^{134}$

Ogni altra considerazione storica, giuridica e scientifica diventa superflua, anche se la risposta ultima alla domanda "chi è l'uomo della Sindone?" non può, comunque, che essere affidata al raziocinio e giudizio di ciascuno, come del resto fa lo stesso Gesù quando chiede ai suoi discepoli: "Ma voi, chi dite che io sia?". ${ }^{135}$

134 B. Barberis, M. Boccaletti, Il caso Sindone...op.cit., p. 277.

135 Marco, 8, 29. 